\title{
Interleukin 35 Activates Intratumor Neovascularization Via Enhanced Secretion of FGF2 in Hepatocellular Carcinoma Through The Recruitment of Neutrophils, and Blocking it Could Facilitate The Efficacy of The PD- 1 Antibody
}

Wei Gan

Zhongshan hospital of fudan university

Mei-Xia Zhang

First Affiliated Hospital of Nanchang University

Jin-Long Huang

Zhongshan Hospital Fudan University

Pei-Yun Zhou

Zhongshan Hospital Fudan University

Cheng Zhou

Zhongshan Hospital Fudan University

Gao Liu

Zhongshan Hospital Fudan University

Yi-Peng Fu

Fudan university

Bao-Ye Sun

Zhongshan Hospital Fudan University

Chu-Yu Jing

Cancer Hospital

Ruo-Yu Guan

Zhongshan Hospital Fudan University

Zhu-Tao Wang

Zhongshan Hospital Fudan University

Jian Zhou

Zhongshan Hospital Fudan University

Jia Fan

Zhongshan Hospital Fudan University

Yong Yi

Zhongshan Hospital Fudan University

Shuang-Jian Qiu ( $\square$ qiu.shuangjian@zs-hospital.sh.cn )

Fudan University https://orcid.org/0000-0003-2056-6127 


\section{Research}

Keywords: hepatocellular carcinoma, interleukin 35, immunotherapy, neutrophils, programmed cell death protein 1

Posted Date: December 21st, 2020

DOI: https://doi.org/10.21203/rs.3.rs-130889/v1

License: (c) (1) This work is licensed under a Creative Commons Attribution 4.0 International License. Read Full License 


\section{Abstract}

Background: Recently, more and more treatment strategies for Hepatocellular carcinoma (HCC) have emerged, but the therapeutic effect is still not satisfactory. This study is aimed to explore the mechanism of Interleukin 35 (IL-35) in promoting the progression of liver cancer and to explore the application value of IL-35 in the treatment of HCC.

Methods: We used clinical tissue microarray (TMA) immunohistochemistry (IHC) to explore the prognostic value of IL-35 expression in patients with HCC. The effect of IL-35 on the function of HCC was explored by functional experiments including wound-healing assay, transwell, cell counting kit-8, cell adhesion assay and endothelial tube formation assay in vitro and mouse xenografts in vivo. And flow cytometry was used to study the effect of IL-35 on infiltrating immune cells in tumor. The molecular mechanism of the function of IL-35 on the progression of HCC was explored by sequencing, ELISA, WB, PCR and other technical means. Finally, through in vivo tumor animal experiments to explore the value of anti-IL-35 antibody and combined with antiPD-1 antibody in the treatment of liver cancer.

Results: High expression of IL-35 in patients with HCC were identified to be associated with poor prognosis. And we have found that IL-35 facilitated tumor progression by affecting neutrophil infiltration, angiogenesis, and CD8+ T-cell infiltration in a mouse model. Additionally, on the one hand C-C motif chemokine ligand 3 (CCL3) has been found to be a key factor mediating the recruitment of neutrophils by IL-35, on the other hand fibroblast growth factor 2 (FGF2) secreting by neutrophil when stimulated by IL-35 was also found to be the core cytokine to promote intratumoral angiogenesis. And IL-35 was also discovered to facilitated the adhesion of tumor to endothelial cells, with neutrophils further enhancing this effect in vitro and vivo. More important, anti-IL-35 antibody was found to be a valid treatment for HCC in xenograft tumor model, and it could give full play to the curative effect of 1:1ه2 when combination therapy with PD-1 antibody.

Conclusion: Our data show that the expression of IL-35 in patients with HCC is an important tumor promoting factor. The application of anti-IL-35 antibody and treatment combined anti-IL-35 antibody with anti-PD-1 antibody have potential therapeutic value in the treatment of liver cancer.

\section{Background}

Hepatocellular carcinoma (HCC), the most common type of primary liver cancer, is the fourth most common malignant tumor and third in mortality in China ${ }^{[1,2]}$. In recent years, radical treatments, such as surgery (hepatectomy and liver transplantation), radiofrequency or microwave ablation of liver tumors have made great progress, resulting in the significant improvement of the therapeutic effect. However, the rate of tumor recurrence and metastasis in the 5 years after operation has been shown to be as high as 60 to $70 \%$, with a lack of effective drugs ${ }^{[3-5]}$. Targeted drug therapy represented by the administration of sorafinib, lovatinib, and regofinib was reported to provide an improvement of only 2.8 months in the overall survival of patients with advanced $\mathrm{HCC}^{[6,7]}$. At present, immunotherapy, including the use of programmed cell death protein 1 (PD1) and programmed cell death protein ligand 1 (PD-L1) antibodies, as well as other immune checkpoint antibodies has attracted much attention. However, the effective rate was shown to be only about 15 to $20 \%$ in patients with HCC, and thus there is an urgent need to find a more effective strategy for the treatment of liver 
cancer $^{[8-10]}$. Fortunately, the combination of immune checkpoint inhibitors and antiangiogenic antibodies has been shown to be extremely effective in the treatment of liver cancer, resulting in revolutionary therapeutic results $^{[11]}$. We thus aimed to explore more effective treatments for HCC.

IL-35 which was first reported in 1997, is a new member of the cytokine IL-12 family, consisting of 2 subunits, $\mathrm{P} 35$ and EBI3 ${ }^{[12,13]}$. It is known to be primarily expressed by Treg and Breg cells, and also secreted by macrophages, dendritic cells (DCs), monocytes, endothelial, and CD4+HLA-G+ cells. It was confirmed that IL35 has a strong immunosuppressive function and plays an important role in inflammation, infection, autoimmune diseases, transplantation immunity, tumor, and other diseases ${ }^{[14-18]}$. It has also been reported that tumor cells, including those from pancreatic cancer, HCC, cholangiocarcinoma, nasopharyngeal carcinoma, breast cancer, and melanoma could also synthesize and secrete IL-35, playing an important role in carcinogenesis and malignant biology ${ }^{[14,19-23]}$. Its specific mechanisms of action have been demonstrated to be the following: (1) promoting tumor cell proliferation and inhibiting tumor cell apoptosis ${ }^{[24]}$; (2) inducing tumor angiogenesis ${ }^{[23]}$; (3) promoting tumor metastasis by increasing the adhesion of tumor cells to endothelial cells and transendothelial migration ${ }^{[25]}$; (4) reversing the epithelial-mesenchymal transition (EMT) of tumor cells to promote tumor colonization in the target organ during metastasis ${ }^{[18]}$; (5) upregulating the expression of immune checkpoint proteins, such as PD-1, Lymphocyte-activation gene 3 (LAG3), and TIM3 in the tumor microenvironment (TME) and promoting the apoptosis of cytotoxic T-lymphocytes (CTL) ${ }^{[26,27] ;}$; 6 ) recruiting myeloid inhibitory cells to form an immunosuppressive network, and reducing the sensitivity of tumor cells to CTL cells ${ }^{[23]}$; and finally (7) promoting the differentiation of neutrophils towards the N2 tumor phenotype by enhancing the secretion of interleukin 6 (IL-6)and Granulocyte Colony-Stimulating Factor (G$\mathrm{CSF})^{[28]}$. Generally speaking, IL-35 is known to directly enhance the proliferation and adhesion of tumor cells. In contrast, it has also been shown to promote tumor angiogenesis and maintain an inhibitory TME by reshaping the local tumor microenvironment. Overexpression of IL-35 has been reported to be closely related to poor prognosis in a variety of tumors, including HCC. Early intervention screening of high-risk patients for overexpression of IL-35 might become a new treatment strategy for the prevention and treatment of the postoperative recurrence and metastasis in patients with HCC. However, at present, the mechanism of IL-35 in HCC is not clear. Therefore, in this study we systematically explored the mechanism of the effect of IL-35 on the malignant biology of liver cancer.

\section{Methods}

\section{Cell lines, mouse xenografts, human samples, and collection of patient clinical data}

LO2 and the HepG2, Huh7, PLC, Hep3B, MHCC97L, MHCC97H, and HCCLM3 HCC cell lines were obtained from the Liver Cancer Institute, Zhongshan Hospital, Fudan University. All cell lines were cultured in a reasonable conditioned media at $37{ }^{\circ} \mathrm{C}$ in a humidified incubator containing $5 \% \mathrm{CO}_{2}$, as previously described.

Male C57BL/6 and BALB/c-nu/nu mice (4-6 weeks old, from Shanghai Institute of Material Medicine, Chinese Academy of Science) were housed under pathogen-free conditions. Animals were cared for in accordance with the guidelines established by the Shanghai Medical Experimental Animal Care Commission. All experimental protocols were approved by the Zhongshan Hospital Research Ethics Committee. All experimental procedures 
involving animals were approved by the Animal Care and Use Committee of Zhongshan Hospital, Fudan University, China.

A total of 360 patients with primary liver cancer who underwent radical resection at Zhongshan Hospital affiliated to Fudan University in 2012 were selected for this study. The obtained samples were confirmed to be HCC based on pathological assessment. Clinicopathological data, such as liver function, alpha-fetoprotein (AFP), carbohydrate antigen 19-9 (CA19-9), carcinoembryonic antigen (CEA), tumor size, number and differentiation, lymph node metastasis, vascular invasion, cancer thrombus formation, and microvascular invasion (MVI) were collected from the medical history and pathological reports of enrolled patients.

\section{IHC and immunofluorescence}

The P35, EBI3, GP130, IL-12 Rß2, P40, and P28 antibodies were used to detect the level of protein expression in tumor tissues using $\mathrm{IHC}$, based on the experiment protocol used in our previous study.

A number of pathology teachers were invited to evaluate the results, and $\mathrm{H}$-scores were used to quantify P35, $\mathrm{EBI} 3, \mathrm{GP130}$, IL12Rß2, and other indexes. According to the calculation score of intensity and area, the intensity of positive staining was divided into 4 grades: 0 , no staining; 1 , weak staining; 2 , moderate staining; and 3 , strong staining. This was divided into 4 levels according to the area of colored cells: $0,<1 \% ; 1,1-25 \% ; 2,26-$ $50 \% ; 3,>50 \%$. Dyeing was divided into the score of the intensity area, with the score of uneven dyeing requiring to be accumulated. The comprehensive score was divided into 4 grades: negative (-), 0 points; weak positive (+), 1-2 points; medium positive $(++)$, 3-4 points; and strong positive $(++)$, 5-9 points. Immune cells, such as CD4+, CD8+, CD34+, CD68+, neutrophils (CD66b+), and FOXP3+ cells were used to evaluate the level of infiltration. Five typical visual fields were randomly selected on the patient chip, and the average value was calculated as the immune cell count of the patient. The cutoff value of the statistical score was calculated using the X-tile software. The results of the chip were independently evaluated by 2 professionals, and any contradictory results were discussed and a joint decision was made.

Immunofluorescence was performed on the HCC cell line, where we explored the expression level of EBI3 and P35. Slides were prepared in the same manner as for IHC before incubation with antibodies. After that, incubation with anti-mouse and anti-rabbit antibodies was performed.

\section{ELISA, WB, coimmunoprecipitation (CO-IP), and quantitative reverse transcription PCR (qRT-PCR).}

Western blotting (WB) was performed as previously described. Briefly, we generated total cell lysates and proteins were separated on SDS-PAGE gels, and then transferred to polyvinylidene difluoride (PVDF) membranes. Membranes were washed and blocked. Next, membranes were incubated with primary and secondary antibodies, detected using enhanced chemiluminescent (ECL) substrate, and processed using the Image Lab software.

Coimmunoprecipitation (CO-IP) was performed as previously described ${ }^{[29]}$. Briefly, both input and IP samples were analyzed by WB using various antibodies at the indicated dilutions.

ELISA kits were used to detect the levels of secretory IL-35, CCL3, and FGF2 in the supernatants of cell cultures according to the manufacturer's instructions. 
Quantitative reverse-transcription PCR was performed using a SYBR PrimeScript reverse-transcription PCR Kit (Takara Bio, Shiga, Japan) in accordance with the manufacturer's instructions. The RT2 profiler array was probed using the Profiler PCR Array System and SYBR green/fluorescein quantitative PCR Master Mix (SABiosciences) in an ABI 7900 sequence analyzer (Applied Biosystems, Carlsbad, CA, USA) in accordance with the manufacturer's protocol.

\section{Wound-healing assay and Transwell Cell Counting Kit-8}

Analyses of cell proliferation were performed using the Cell Counting Kit-8 (CCK-8; Dojindo). Wound-healing assay and noncoated transwell chambers (BD Pharmingen) were used to assess the migratory ability of cells. The invasion ability of cells was assessed using Matrigel-coated transwell chambers (BD Pharmingen).

\section{Cell adhesion assay and endothelial tube formation assay}

Human umbilical vein endothelial cells (HUVECs) were cultured with ECM, in slices, and allowed to reach full confluence. Then, the monolayers were stained with a CytoPainter Cell Tracking Staining Kit (Abcam) and inserted into a flow chamber. HCC cells were labeled with Calcein AM (Donjindo) according to the manufacturer' s instructions. Briefly, $1 \times 10^{5}$ labeled HCC cells were added to HUVEC cells and cocultured for 2 $\mathrm{h}$ at $37^{\circ} \mathrm{C}$ in a cell incubator. After that, the culture medium was removed, and the unattached tumor cells were washed twice with PBS before being replaced with complete culture medium. Adhered tumor cells on the monolayers were imaged using a fluorescence microscope and their average number was calculated in 5 random fields.

In vitro endothelial tube formation assays were performed using collagen gel. First, Matrigel was kept melt on ice, and then $250 \mathrm{~mL}$ of melted matrix glue was gently poured into each hole of a precooled 24-well plate to avoid bubbles. The plate was then placed into a $37^{\circ} \mathrm{C}$ incubator for 35 min for solidification. HUVECs $(8 \times$ $10^{4}$ cells/well in a 24-well plate) were cultured on the surface of collagen gel using endothelial cell medium (ECM) and other conditioned media. The total tube length, the number of branch points, and the number of loops in the tube-like structures were quantified in 24 fields per group.

\section{Statistical analysis}

Statistical analyses were performed using the IBM SPSS and GraphPad Prism. Each experiment was performed in triplicate, and values are presented as the mean \pm SD, unless otherwise stated. The variance between the groups was statistically compared. The distributions of both the overall survival (OS) and recurrence-free survival (RFS) were depicted using the Kaplan-Meier method and analyzed by the log-rank test. Univariate and multivariate analyses for prognostic factors were based on the Cox proportional hazard model. $\mathrm{P}<0.05$ was considered significant.

\section{Results}

Co-expression analysis of P35 and EBI3 subunits in hepatocellular carcinoma could be used as a proxy for the level of IL-35. 
At present, there is no high-quality specific antibody for IL-35. So in order to investigate the level of IL-35, we detected the expression of P35 and EBI3 in continuous tissue sections of HCC samples, which has been reported to be a reliable method in other research studies ${ }^{[22,25]}$. As IL-12, IL-27, and IL-35 are known to share common subunits, we detected all 4 subunits, namely P35, EBI3, P40, and P28 in TMA of patients with HCC. The P35 and EBI3 proteins were observed to be mainly located in the cytoplasm, widely expressed in tumor cells, as well as in stromal cells. Whereas the expression of P35 and EBI3 was demonstrated to be lower in paracancerous compared with cancerous tissues of the same patient, we found that the expression level of

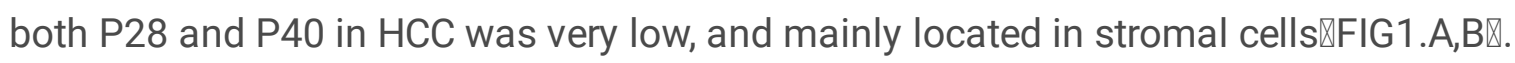

The proportional structure expression map of EBI3 and P35 was shown to be highly similar, but obviously different from that of P28 and P40. We further observed that the staining scores of EBI3 and P35 were strongly correlated $(r=0.698, P<0.001)$. However, there was a poor association between EBI3 and P28 $(r=$ $0.08, P<0.05)$, as well as between $P 35$ and $P 40(r=0.042, P<0.001)(F I G 1 . D)$.

Furthermore, we used the HCCLM3, MHCC97H, SMMC-7721, and HUH7 cell lines to carry out cellular immunofluorescence experiments. We accordingly found that P35 and EBI3 were mainly expressed in the cytoplasm, and not in the nucleus. The expression intensity of P35 and EBI3 was shown to be basically the same, with the spatial expression sites basically overlapping. The expression intensity of these 2 proteins was found to be higher in HCCLM3 and MHCC97H, but low in SMMC-7721 and HUH7 (FIG1.C). In order to eliminate the effect of the cosubunits of IL-12 and IL-27 on the detection of IL-35, we used the CO-IP technique to study the structural relationship of the 4 subunits in 4 cases of HCC. The P35 antibody was demonstrated to successfully immunoprecipitate EBI3 but rarely bound to P40 and P28. Similarly, the same phenomenon was observed in CO-IP experiments in the MHCC97H and HCCLM3 cell lines (FIGS1.B).

\section{Overexpression of IL-35 in hepatocellular carcinoma was an independent risk factor for prognosis.}

We then analyzed the baseline characteristics of the 360 patients with HCC. Our study included 202 men (56.1 $\%)$ and 158 women (43.9\%), with an average age of $54.36 \pm 11.038(26-85)$ years and a median age of 54 years. The average overall survival (OS) time was $51.87 \pm 0.996$ months, and the median OS was $49 \pm 7.19$ months. The average recurrence-free survival (RFS) time was $41.91 \pm 1.27$ months, and the median RFS was $22.0 \pm 1.837$ months (Table1.).

The IHC staining score ++/+++ was stratified as high expression. The high expression of both P35 and EBI3 was defined as the IL-35 high expression group (41.6\%), whereas others were classified as the IL-35 low expression group (58.4\%) (FIG1.D). Significant correlations were found between the high expression of IL-35 and advanced Barcelona clinic liver cancer (BCLC) stage. Moreover, we also found that the level of serum alpha-fetoprotein (AFP) was significantly increased in the IL-35 overexpression group (637.45 \pm 32.8 vs 212.47 $\pm 18.9 \mathrm{ng} / \mathrm{mL}, \mathrm{P}<0.05$ ). In addition, we noted that overexpression of IL-35 was closely related to an increased prevalence of portal vein tumor thrombus (PVTT), microvascular invasion (MVI), and large tumor size $(\mathrm{P}<$ 0.001). Multivariate analysis showed that overexpression of IL-35 was an independent risk factor for both OS $(\mathrm{HR}=1.947 ; 95 \% \mathrm{Cl}, 1.046-3.624, \mathrm{P}=0.035)$ and $\mathrm{RFS}(\mathrm{HR}=2.442 ; 95 \% \mathrm{Cl}, 1.459-4.088)$ (Table2.). Therefore, the expression of IL-35 in HCC was considered an important reference index for judging prognosis. 
Binding of cytokines to receptors is known to be an important link for the functional role cytokines play in cells. After binding to receptors, IL-35 is known to activate the intracellular signal transduction pathway. Therefore, it is of great significance to explore the expression of the GP130 and IL-12 R 32 receptors of IL-35 in HCC. At present, there has been no report on the expression of receptors of IL-35 in HCC. We found that the expression of GP130 and IL-12 Rß2 in HCC was closely related ( $r=0.39, P=0.023)$ (FIG2B). Based on the expression of IL-35 and its receptors in HCC, we divided the 360 patients into 4 groups: IL-35R (+) IL-35 (high); IL-35R (+) IL-35 (low); IL-35R (-) IL-35 (high); and IL-35R (-) IL-35 (low). We found that patients in the IL-35R (+) IL-35 group (high) had the worst prognosis $(P<0.001)$, thus supporting the hypothesis that IL-35 facilitated the progression of HCC by directly acting on tumor cells in an autocrine or paracrine manner(FIG2C).

\section{Overexpression of IL-35 in patients with hepatocellular carcinoma was closely related to the infiltration of neutrophils and $\mathrm{CD}^{+}$in tumor microenvironment.}

We further explored the relationship between the level of IL-35 and TME in HCC. Our results showed that the infiltration of neutrophils in HCC tissues with high levels of IL-35 was significantly higher than that in the low expression group (5.31 vs. $14.80 \pm 1.34$, $P<0.001)$. In addition, we found that the number of infiltrated CD8+ Tcells in tissues with high levels of IL-35 was significantly decreased (34.55 \pm 2.758 vs. $56.61 \pm 3.53, \mathrm{P}<$ 0.001 ). Importantly, the number of microvessel density (MVD) labeling by CD34 in patients with overexpression of IL-35 was demonstrated to be significantly increased (86.63 \pm 4.789 vs. $56.54 \pm 2.308$, P < 0.001 ). Additionally, the number of neutrophils infiltrating the tumor was shown to be positively correlated with MVD $(r=0.301, P<0.001)$, suggesting that neutrophil infiltration might be an important factor in tumor angiogenesis(FIG2A-C).

\section{IL-35 facilitated tumor progression by affecting neutrophil infiltration, angiogenesis, and CD8+ T-cell infiltration in a mouse model.}

Based on the expression of IL-35 in HCC cell lines, we selected the HCCLM3 and MHCC97H cell lines, which exhibited a high expression level to construct IL-35 knocked-down cell lines. Whereas the HUH7 and SMMC7721 cell lines, which showed a low expression level of IL-35 were selected to construct IL-35 overexpressing cell lines. We also constructed IL-35 overexpressing and knocked-down Hepa1- 6 cells. These HCC cell lines were also used to detect the expression of the 2 subunits of the IL-35 receptor, GP130 and IL-12 R 2 . These were observed to be structural foundations for liver cancer cells in affecting the function of tumor cells through autocrine IL-35(FIGS1A).

First, we used the CCK8 transwell and wound healing to test the effect of IL-35 on HCC cells. Our results showed that IL-35 had no significant direct effects on the proliferation and migration of HCC cells in vitro. Surprisingly, we found that the formation rate of the subcutaneous tumor in the IL-35 overexpressing cell line in nude mice was significantly faster than that in the control group (volume $\mathrm{mm}^{3}: 1186.81 \pm 83.53$ vs. 612.82 $\pm 73.49, \mathrm{P}<0.001$, tumor mass $\mathrm{g}: 1.17 \pm 0.11$ vs. $0.63 \pm 0.09, \mathrm{P}<0.001$ ), with the tumor growth rate being significantly slowed down in the knocked-down group (volume $\mathrm{mm}^{3}$ : $495.48 \pm 53.42$ vs. $882.61 \pm 73.25, \mathrm{P}<$ 0.001 , tumor mass g: $0.62 \pm 0.07$ vs. $1.11 \pm 0.18, P<0.001)$ (FIG3). We also observed the same phenomenon in the immunocompetent C57BL/6 mouse model when exploring the effect of different expression levels of IL-35 
on the growth of subcutaneous tumors(FIG4). Therefore, we speculated that IL-35 might promote the progression of liver cancer by affecting TME.

Meanwhile, we found that the infiltration of neutrophils ( $169 \pm 47$ vs. $62 \pm 15, P<0.001)$, as well as the number of MVD (149 \pm 39 vs. $33 \pm 21, P<0.001)$ were both significantly increased in immunodeficient or immunocompetent mouse models injected with tumor cells overexpressing IL-35. In the IL-35 knocked-down

group, the number of neutrophils infiltrating the tumor ( $31 \pm 12 \mathrm{vs.} 189 \pm 43, P<0.001)$, and the amount of MVD (43 \pm 21 vs. $125 \pm 34, P<0.001$ ) were shown to be significantly decreased(FIG3B). Thus, we assumed that IL-35 could promote intratumoral neovascularization by recruiting the infiltration of neutrophils in tumors. To test this hypothesis, we used a LY6G neutrophil antibody to deplete neutrophils in an IL-35-overexpressing subcutaneous tumor, and found that depleting neutrophils could significantly reverse the promoting effect of the overexpression of IL-35(FIG4D).

In addition, we also found that the number of infiltrating CD8+ T-cells was significantly decreased in the overexpression group, whereas it was significantly increased in the knocked-down group.

\section{IL-35 promoted neutrophil infiltration by increasing the expression of CCL3 in vitro}

We used a transwell assay to verify the effect of the HCC-related expression of IL-35 on neutrophil chemotaxis. The chemotactic effect of the IL-35-KD conditioned medium (CM) on neutrophils was shown to be decreased by $64.5 \%$ and $56.3 \%(P<0.05)$, whereas the overexpression in $\mathrm{CM}$ increased by 3.97 and 4.67 times, respectively $(P<0.05)$. However, we noted that the recombinant IL-35 (rlL-35) had no significant effect on neutrophil chemotaxis $(P>0$ 05). These results showed that HCC-related IL-35 did not directly affect neutrophil infiltration(FIG5A).

We then aimed to further explore the pathway through which IL-35 affects the chemotaxis of neutrophils. After comparing the results of IL-35 positive related genes and sequencing in the TCGA database, we found that the expression of neutrophil-related chemokine genes was significantly increased after overexpression of IL-35(FIGS2A). We further found that following overexpression of IL-35, the intracellular levels of the CCL3 protein were significantly increased, whereas after knocking down IL-35, the intracellular levels of the CCL3 protein were shown to be significantly decreased. We further confirmed that CCL3 was significantly increased in IL-35-overexpressing patients ( $\mathrm{P}<0.012, \mathrm{r}=0.431$ ) (FIG5B). In order to verify whether IL-35 chemotactically affected neutrophils through the expression of CCL3, we carried out a CCL3 antibody block test. We accordingly discovered that CCL3 could significantly enhance the chemotactic effect on neutrophils, as the CCL3 antibody intervention test was demonstrated to significantly reduce the chemotactic effect of CM on neutrophils(FIG5A). In summary, we found that IL-35 could promote the chemotactic effect of neutrophils by promoting the expression of CCL3 in HCC.

\section{IL-35 stimulated neutrophil secretion of FGF2 to promote angiogenesis}

To illustrate the roles and underlying mechanism of IL-35 in tumor angiogenesis, we carried out a tube formation experiment in vitro. First, we stimulated HUVEC endothelial cells with rlL-35 or CM from IL-35overexpressing or knocked-down cells, and found that the tube formation rate did not significantly change. This suggested that IL-35 did not directly stimulate the formation of vascular endothelium. Considering that 
accumulation of neutrophils in HCC tissues has been reported to increase the production of angiogenesis factors and facilitate microvessel formation, we speculated that IL-35 might indirectly affect tumor angiogenesis by stimulating neutrophils. To further assess this hypothesis, we stimulated HUVECs with CM from the coculturing of neutrophils and HCC cells. We found that CM from neutrophils cocultured with IL-35overexpressing HCC cells could enhance tube formation (tube density: $212 \pm 31$ vs. $141 \pm 19, \mathrm{P}<0.0024$; tube branch: $365 \pm 27$ vs. $238 \pm 24, \mathrm{P}<0.001$ ). Conversely, the CM from IL-35 knocked-down HCC cells cocultured with neutrophils could significantly inhibit the tubule formation of endothelial cells (tube density: $119 \pm 19$ vs. $169 \pm 23, \mathrm{P}<0.0056$; tubule branch: $229 \pm 24$ vs. $315 \pm 32$, $\mathrm{P}<0.0013)$. (FIG5C) These results demonstrated that IL-35 stimulated neutrophils to produce angiogenesis factors.

To further explore this, we isolated neutrophils from patients with HCC, stimulated them with human IL-35, and then sequenced them. Our sequencing results were subjected to $G O$ and KEGG analysis, where it was revealed that the expression of genes related to angiogenesis and adhesion factors in neutrophils was significantly increased. The KEGG pathway enrichment map showed that after neutrophils were stimulated by IL-35, the pathways of epidermal growth factor receptor (EGFR) and vascular endothelial growth factor (VEGF) were significantly activated. The FGF2 protein was demonstrated to be the most significantly elevated angiogenic factor, with the expression of the FGFR3 and FGFR4 receptors of FGF2 being also increased by 574 and 65 times, respectively (FIGS2B,FIG6A). This finding suggested that there might be a mechanism by which IL-35 stimulates the positive feedback secretion of FGF2 by neutrophils. This was further confirmed by WB analysis.

To explore whether FGF2 plays a decisive role in mediating IL-35 to promote angiogenesis, we performed blocking experiments. When anti-IL-35 and FGF2 neutralizing antibodies were used, the tube formation rate was shown to be significantly abrogated. Furthermore, after IL-35 knocked-down HCC cells were cocultured with neutrophils in the presence of rlL-35, the tube formation rate was demonstrated to be significantly elevated; however, when anti-FGF2 neutralizing antibody was added to the above CM, tubule formation was blocked.(FIG6B)

\section{IL-35 facilitated the adhesion of tumor to endothelial cells, with neutrophils further enhancing this effect in vitro.}

We also found that IL-35 could enhance the adhesion of HCC cells to HUVECs in vitro. Following the overexpression of IL-35, the adhesion rate of SMMC-7721 or HUH7 cells to endothelial cells was demonstrated to be significantly increased. In contrast, knocking down IL-35 strongly inhibited the number of HCCLM3 or $\mathrm{MHCC} 97 \mathrm{H}$ cells adhered to endothelial cells. At the same time, we also found that when neutrophils were added to overexpressing IL-35 trials, the number of SMMC-7721 or HUH7 cells adhered to endothelial cells was increased by $46 \%$ and $54 \%$, respectively $(P<0.001)$. However, there was no significant change observed in the adhesion of tumor cells cocultured with neutrophils in the knocked-down group $(P>0.05)$. These results showed that IL-35 could increase the adhesion of hepatocellular carcinoma cells to endothelial cells, and neutrophils could further enhance this effect. In addition, we further verified this finding using the Hepa1-6 mouse HCC cell line (FIG7A).

The lung metastasis model of the tail vein in nude mice further showed the promoting ability of IL-35 and neutrophils.

Page 10/31 
In the in vivo experiment, we first labeled tumor cells with a fluorescent dye. Following the intravenous injection of HCC cells into the tail vein of nude mice, the remaining tumor cells in the lung tissue were observed using a fluorescence tracer. We found that there was no significant difference in the retention of tumor cells in each group at $30 \mathrm{~min}$; however we observed a significant difference in the number of tumor cells stranded in lung tissue $24 \mathrm{~h}$ later. The number of retained tumor cells in the coinjection group ( $279 \pm 53$ )was significantly higher than that in the overexpression $(103 \pm 31)$ and control $(62 \pm 21)$ groups $(P<0.001)$, whereas the number of tumor cells retained in the overexpression group was also significantly higher than that in the control group. We observed that in the coinjection group, a large number of tumor cells adhered directly to neutrophils. We collected gross specimens of lung tissue, and found that the number of metastatic nodules after injecting HUH7-OE was significantly higher than that in the control group (FIG7A). After simultaneous injection of mixed cells of neutrophils and $O E$ strains, the number of pulmonary metastatic nodules was demonstrated to be further increased. This number was significantly higher than that of the OE stable lung metastasis model, consistent with the results of $\mathrm{HE}$ staining of lung metastasis nodules. Meanwhile, we found that the rate of lung metastasis was significantly decreased when IL-35 was knocked down (FIG7B).

\section{Anti-IL-35 neutralizing antibody enhanced the efficacy of PD-1 antibody}

The combined use of drugs is an important way to explore better treatments of liver and other cancers. Therefore, we aimed to explore whether an IL-35 antibody could enhance the effect of the administration of the PD-1 antibody in the treatment of HCC.

We found that in the subcutaneous tumor model of immunocompetent mice recipients bearing Hepa1-6 cells, tumor growth was slightly suppressed after treatment with either an anti-IL-35 or a PD-1 neutralizing antibody. However, we observed more dramatic and durable responses, compared with the responses in the control treatment, when the anti-PD-1 antibody was combined with the anti-IL-35 neutralizing antibody(FIG8A). We also constructed an in vivo model using the IL-35-OE Hepa1-6 cell line, followed by the administration of both the anti-PD-1 and anti-IL-35 antibodies. Our results showed that the anti-PD-1 antibody combined with treatment with the anti-IL-35 antibody could reverse the increased tumor growth induced by IL-350E Hepa1-6 cells in C57BL/6 mice (FIGS3).

As shown in Figure8B-C, the infiltration of CD8+ T-cells was increased after treatment with the IL-35 and PD-1 antibodies $(P<0.001)$. In contrast, we found that neutrophil infiltration was decreased after treatment with the IL-35 antibody $(P<0.001)$, whereas no effect was observed in neutrophil infiltration after administration of the PD-1 antibody. Nevertheless, we did not observe any significant difference in other cells, including macrophages and Treg cells. Immunohistochemical analysis showed that treatment with the PD-1 and IL-35 antibodies could increase the infiltration of CD8+ T-cells in the tumor, with the combined treatment group being shown to further increase the infiltration of CD8+ T-cells compared with the single treatment group $(P<0.001)$. The neutrophil infiltration in the IL-35 antibody group and the combined treatment group was observed to be significantly lower than that in the control group $(P<0.001)$; however, there was no significant difference shown in neutrophil infiltration between the 2 groups. The PD- 1 antibody treatment group was also shown to have no effect on neutrophil infiltration.

\section{Discussion}


Cytokines, including interleukin, chemokine, and growth factors, are small molecules synthesized and secreted by immune cells (monocytes, macrophages, NK cells, and other cells) and some nonimmune cells (endothelial cells, fiber cells, etc.). Cytokines are known to directly act and participate in the proliferation and metastasis of tumor cells, and to indirectly participate in the occurrence and development of tumors through the regulation of TME. Functionally, cytokines can be divided into 2 categories: antitumor and tumor-promoting. The former is represented by IL-2, tumor necrosis factor (TNF), and interferon (IFN), whereas the latter is represented by transforming growth factor (TGF), IL-10, VEGF, and FGF. As a special member of the IL-12 family, IL-35 has been reported to play an important role in tumor immunosuppression. We found that IL-35 promoted intratumoral neovascularization by recruiting neutrophils and reshaping the immune microenvironment to promote the progression of liver cancer. In addition, the combination of anti-IL-35 and anti-PD-1 antibodies was demonstrated to play a synergistic role in anticancer activity.

We found that overexpression of IL-35 in HCC was an independent risk factor for overall survival and recurrence. The GP130 and IL-12 R 2 receptors were also found to be expressed in HCC tissues, indicating a structural basis for the paracrine and autocrine function of IL-35, consistent with previous reports ${ }^{[22,30]}$. We further combined the expression levels of IL-35 and its receptors in HCC tissues, and found that patients with high expression of IL-35 and its receptors in tumor tissues had the worst prognosis, indicating that IL-35 expressed in HCC might activate the receptors of tumor cells through an autocrine pathway to promote the progression of liver cancer. However, no significant correlations were noted between the overexpression of IL35 and advanced BCLC stage, higher levels of serum AFP, larger tumors, and higher risk of PVTT and MVI, thus suggesting a significant role of IL-35 in the progression and metastasis of HCC.

Interestingly, although the results of CCK8, transwell, and wound healing assays were negative in vitro, we found that in vivo, elevated IL-35 could significantly promote tumor growth, whereas the downregulation of the expression of IL-35 was significantly inhibited in both immunocompetent and immunodeficient mouse models. It has also been previously reported that low expression of miR-28-5p in hepatocellular carcinoma cell lines did not affect the proliferation and invasion of tumor cells in vitro, but could promote the tumor secretion of IL-34 known to lead to the recruitment and transformation of tumor-related macrophages to promote the metastasis of hepatocellular carcinoma cells ${ }^{[31]}$. Wang et al. reported that melanoma-derived IL-35 could promote the growth of subcutaneous tumors in immunocompetent mice by recruiting myeloid inhibitory cells to infiltrate the $\mathrm{TME}^{[23]}$. A study of pancreatic cancer also found that tumor-derived IL-35 could promote tumor growth by recruiting monocytes to infiltrate the tumor ${ }^{[20]}$. Therefore, we speculated that IL-35 might also indirectly affect and promote tumor progression by modifying TME.

By studying the TME of HCC tissue samples, we found that the local infiltration of neutrophils and MVD were significantly increased, whereas that of CD8+ T-lymphocytes was significantly decreased in tumors with high expression of IL-35. In in vivo experiments, we also found that IL-35 was closely related to neutrophil infiltration and intratumoral angiogenesis, suggesting that the increased expression of IL-35 was related to local immune tolerance and tumor angiogenesis in HCC. Although neutrophils are known to play an important role in innate immunity, following their recruitment by tumor cells, N2 neutrophils have been reported to promote tumor growth and metastasis by stimulating tumor angiogenesis. Our study also showed that there was a significant positive correlation between neutrophils and angiogenesis in HCC, and there was a

Page $12 / 31$ 
significant increase in angiogenesis and neutrophil infiltration in HCC tissues with high expression of IL-35. These findings suggested that there might be a complex interaction between the level of IL-35 and neutrophil infiltration and angiogenesis in hepatocellular carcinoma. The factors that affect the formation of blood vessels in tumors are complex. For instance, angiogenic factors could directly stimulate tumors to form neovascularization. However, tumor angiogenesis could also be indirectly affected by the recruitment of neutrophils, macrophages, Treg cells, stellate cells, and other interstitial cells known to affect TME. It has been reported that IL-35 could also affect the secretion of CXCL1 and CXCL 8 by monocytes and promote angiogenesis in pancreatic cancer ${ }^{[20]}$. Wang et al. reported that IL-35 promoted tumor angiogenesis through macrophages in melanoma ${ }^{[23]}$. We found that both direct stimulation of rIL-35 and IL-35- overexpressing or knocked-down CM had no significant effect on microvessel formation, indicating that the tumor did not directly stimulate the formation of vascular endothelium by secreting IL-35. Therefore, we considered that tumor secretion of IL-35 might promote intratumoral angiogenesis through indirect mechanisms.

Tumor-related neutrophils are known to play an important role in the occurrence and development of liver cancer and have been shown to be closely related to tumor angiogenesis. It has been reported that neutrophils (mainly N2) could participate in the reconstruction of the tumor extracellular matrix by secreting matrix metalloproteinases (MMPs), NE, and cathepsin G, thus promoting tumor metastasis ${ }^{[32,33]}$. Tumor cells have been shown to recruit a large number of neutrophils to infiltrate tumor tissues by secreting the CXCL8 chemokine. These infiltrating neutrophils are known to interact with adhesion molecules, such as ICAM1 on the surface of tumor cells to enhance the adhesion and metastasis of tumor cells to endothelial cells ${ }^{[25]}$. Neutrophils in breast cancer were demonstrated to bind to circulating tumor cells (CTCs) entering the circulatory system through vascular cell adhesion protein 1 (VCAM1) to promote metastasis of breast cancer cells $^{[32]}$. Neutrophils have also been shown to directly stimulate tumor proliferation and metastasis by releasing the extracellular capture network (NET), containing chromatin, granule proteins, matrix metalloproteins, and NE. In particular, NET is rich in active protein components, and thus can provide a favorable local microenvironment for tumor cells with transvascular metastasis, recruit tumor cells to form cell clusters, and inhibit the apoptosis of tumor cells. At the same time, it could enhance the adhesion between tumor and vascular endothelial cells ${ }^{[34,35]}$. Zou et al. found that IL-35 could indirectly promote neutrophil polarization to $\mathrm{N}^{[28]}$. We found that IL-35 was associated with neutrophil infiltration, indicating that IL-35 might promote intratumoral angiogenesis through neutrophils. We further found that IL-35 could significantly affect the expression of CCL3 and promote neutrophil infiltration. It should be mentioned that CCL3 is an important chemokine in neutrophils. We further found that IL-35 could promote vascular catheterization and intratumoral angiogenesis by promoting the secretion of the FGF2 angiogenic factor by neutrophils. Moreover, IL-35 was demonstrated to stimulate the expression of the FGFR3 and FGFR4 neutrophil FGF2 receptors, indicating that there might be a positive feedback loop in TME regarding the IL-35-stimulated secretion of neutrophil FGF2. Briefly, FGF2, secreted by neutrophils, is known to be an important cytokine in the promotion of vascular growth. It has been reported that neutrophils infiltrated at metastasis sites could promote intratumor angiogenesis by secreting FGF2, and could thus also promote tumor metastasis ${ }^{[36]}$.

It is interesting that IL-35 could significantly reduce the infiltration of CD8+ T-cells in tumor tissues in an immunocompetent mouse model, a finding that was in accordance with the phenomenon observed in the TMA of HCC patients, suggesting that IL-35 might have an inhibitory effect on tumor immunity. Liver cancer cells 
might also secrete IL-35 to reduce the infiltration of CD8+ T-cells in order to create an immunosuppressive microenvironment, thus inducing tumor immune escape. It has also been reported that IL-35 secreted by tumors did not directly affect the differentiation and function of CD8+ T-cells in melanoma. However, tumor cells have also been reported to stimulate the enhanced expression of the GP130 receptor of IL-35 to reduce their sensitivity to CD8+ T-cell killing, so as to escape immune surveillance and promote tumor growth ${ }^{[23]}$.

Tumor immunotherapy represented by therapy with PD-1/PD-L1 antibodies is a hot spot of tumor immunotherapy in recent years. So far, it has been reported to achieve good results in a variety of tumors, including hepatocellular carcinoma, and has received extensive attention. At present, there are more than 3000 clinical trials evaluating single drugs and combined treatments of immune checkpoints. Several studies have shown that the curative effect of the PD-1 antibody combined with chemotherapy, targeted drugs, and multiple immune checkpoint drugs was better than that of the administration of a single drug. The effect of getting twice the result with half the effort was obtained ${ }^{[37-42]}$. It should be noted that the therapy with PD-1 or PD-L1 antibody is known to be effective mainly through the immunological mechanism playing the antitumor role; however, its efficacy has certain limitations and therefore needs to be combined with other antitumor drugs in order to produce improved results. Recently, a number of studies have shown that immune checkpoint inhibitors combined with antivascular-targeting drugs or antibodies could achieve a breakthrough effect, giving full play to the curative effect of 1:1 $>2$. More optimistically, we found that the IL-35 antibody could enhance the efficacy of PD-1. The possible reasons behind this might be the following: (1) the IL-35 antibody might reduce the inhibitory effect of IL-35 on lymphocytes; (2) the IL-35 antibody could enhance the infiltration of lymphocytes and reduce the infiltration of neutrophils. Related studies have shown that the number of neutrophils infiltrating tumors could promote the progression of liver cancer by secreting C-C motif chemokine ligand 2 (CCL2) and CCL17 to recruit macrophages and Treg cells ${ }^{[31]}$. Blocking neutrophils with the IL-35 antibody could also reduce the recruitment of macrophages and Treg cells and reduce the inhibitory effect on CD8+ T-cells; (3) blocking IL-35 could block neutrophil-mediated intratumoral angiogenesis and further promote the efficacy of the PD-1 antibody. In related studies of hepatocellular carcinoma, it was found that the PD-L1 of neutrophils near the cancer was significantly increased ${ }^{[43]}$; (4) the IL-35 antibody might block the inhibitory effect of PD-L1 expressed on neutrophils on CD8 + T-cells; (5) anti-IL-35 therapy could inhibit intratumoral angiogenesis, reverse the immunosuppressive microenvironment, and enhance the efficacy of the therapy with PD-1 antibody. Besides, therapy with PD-1 antibody could improve the lethality of immune cells, and could also inhibit intratumoral angiogenesis, both of which complement each other and promote each other to form a more powerful therapeutic effect.

Although we found that IL-35 affected the progression of liver cancer, there are still many limitations in this study. First, the IL-35 antibody is an anti-single subunit antibody, and it is difficult to fully assess the blocking effect of IL-35; hence we could not rule out the possibility of blocking the effect of IL-12 or IL-27. Second, IL-35 is a widely secreted cytokine. The use of an IL-35 antibody could not only affect IL-35 secreted by HCC but also IL-35 secreted by other cells, including Tregs. Finally, the clinical samples came from a retrospective single-center study, and as such verification of these clinical data require an extended study.

\section{Conclusions}


Our data show that the expression of IL-35 in patients with HCC is an important tumor promoting factor. And we have found that IL-35 facilitated tumor progression by affecting neutrophil infiltration, angiogenesis, and CD8+ T-cell infiltration in a mouse model. Additionally, on the one hand CCL3 has been found to be a key factor mediating the recruitment of neutrophils by IL-35, on the other hand FGF2 secreting by neutrophil when stimulated by IL-35 was also found to be the core cytokine to promote intratumoral angiogenesis. The application of anti-IL-35 antibody and treatment combined anti-IL-35 antibody with anti-PD-1 antibody have potential therapeutic value in the treatment of liver cancer.

\section{Abbreviations}

APF:alpha fetal protein, CA19-9:carbohydrate antigen 19-9, CEA:carcinoembryonic antigen, TBIL:total bilirubin,ALB:albumin,BCLC:Barcelona Clinic Liver Cancer staging system, HCC:Hepatocellular carcinoma, TMA: tissue microarray, PD 1:programmed cell death protein 1, PD-L1:programmed cell death protein ligand 1, DCs:dendritic cells, EMT:epithelial-mesenchymal transition, TME:tumor microenvironment, CTL:cytotoxic Tlymphocytes, IHC:immunohistochemistry, CO-IP:Coimmunoprecipitation, HUVECs:Human umbilical vein endothelial cells, OS:overall survival, RFS:recurrence-free survival, PVTT:portal vein tumor thrombus , MVI:microvascular invasion, MVD:microvessel density, CCL3:C-C motif chemokine ligand 3, EGFR:epidermal growth factor receptor, VEGF:vascular endothelial growth factor, FGF2:fibroblast growth factor 2, TNF:tumor necrosis factor, IFN:interferon, TGF:transforming growth factor, CTCs:circulating tumor cells, VCAM1:vascular cell adhesion protein 1, NET:extracellular capture network

\section{Declarations}

\section{Acknowledgements}

Not applicable

\section{Authors' contributions}

Wei Gan performed the in vivo and cellular experiments and wrote the manuscript. Meixia Zhang analyzed the data. Jinglong Huang and Peiyun Zhou discussed and participated in the data collection analysis. Cheng Zhou collected data and performed some statistical analysis. Gao Liu, Baoye Sun, Ruoyun Guan, Yipeng Fu and Chuyu Jing Zhutao Wang helped to design the experiments. Yong Yi, Shuang-jian Qiu, Jia Fan and Jian Zhou designed the manuscript, evaluated the results, and revised the manuscript.

\section{Funding}

This project is supported by the 67th batch of general grants from the Chinese Postdoctoral Science Foundation, (NO. KLF152042), the National Natural Science, Foundation of China, (NO. 82072677), the National Natural Science, Foundation of China, (NO. 81772510); National Key Research and Development Program of China (NO. 2017YFC0908101 and NO. 2017YFC0908102); Research Programs of Science and Technology Commission Foundation of Shanghai (NO. 18XD1401100) and Shanghai Municipal Key Clinical Specialty. 
Availability of data and materials

The datasets used during the current study is available from the corresponding authors upon reasonable request.

\section{Ethics approval and consent to participate}

Animal experiment protocols were approved by the ethics committee of the zhongshan Hospital of Fudan University. And the study protocol was approved by the ethics committee of the Zhongshan Hospital of Fudan University

\section{Consent for publication}

Not applicable.

\section{Competing interests}

This paper has not been submitted elsewhere for consideration of publication. The authors certify that they have participated sufficiently in the work to take public responsibility for the appropriateness of the experimental design and method, and the collection, analysis, and interpretation of the data. All authors have reviewed the final version of the manuscript and approved to submit it to your journal. There is no conflict of interest of any authors in relation to the submission.

\section{Authors' information (optional)}

1. Department of Liver Surgery and Transplantation, Zhongshan Hospital, Fudan University, Liver Cancer Institute, Fudan University, Key Laboratory for Carcinogenesis \& Cancer Invasion, The Chinese Ministry of Education, Shanghai, People's Republic of China

2. Department of Gastroenterology, The First Affiliated Hospital of Nanchang University, 17 yongwaizheng street, nanchang, 330006, jiangxi, people's republic of china

3. Department of Breast Surgery, The Obstetrics \& Gynecology Hospital of Fudan University, Shanghai, People's Republic of China

4. Fudan University Shanghai Cancer Center, 270 Dong-An Road, Shanghai, 200032, People's Republic of China.

\section{References}

1. Forner A, Reig M, Bruix J. Hepatocellular carcinoma. Lancet. 2018. 391(10127): 1301-1314.

2. Forner A, Llovet JM, Bruix J. Hepatocellular carcinoma. Lancet. 2012. 379(9822): 1245-55.

3. Ishizawa T, Hasegawa K, Aoki T, et al. Neither multiple tumors nor portal hypertension are surgical contraindications for hepatocellular carcinoma. Gastroenterology. 2008. 134(7): 1908-16.

4. Reig M, Mariño Z, Perelló $C$, et al. Unexpected high rate of early tumor recurrence in patients with HCVrelated HCC undergoing interferon-free therapy. J Hepatol. 2016. 65(4): 719-726. 
5. Singal AG, Pillai A, Tiro J. Early detection, curative treatment, and survival rates for hepatocellular carcinoma surveillance in patients with cirrhosis: a meta-analysis. PLoS Med. 2014. 11(4): e1001624.

6. Llovet JM, Ricci S, Mazzaferro V, et al. Sorafenib in advanced hepatocellular carcinoma. N Engl J Med. 2008. 359(4): 378-90.

7. Personeni N, Pressiani T, Santoro A, Rimassa L. Regorafenib in hepatocellular carcinoma: latest evidence and clinical implications. Drugs Context. 2018. 7: 212533.

8. El-Khoueiry AB, Sangro B, Yau T, et al. Nivolumab in patients with advanced hepatocellular carcinoma (CheckMate 040): an open-label, non-comparative, phase 1/2 dose escalation and expansion trial. Lancet. 2017. 389(10088): 2492-2502.

9. Zhu AX, Finn RS, Edeline J, et al. Pembrolizumab in patients with advanced hepatocellular carcinoma previously treated with sorafenib (KEYNOTE-224): a non-randomised, open-label phase 2 trial. Lancet Oncol. 2018. 19(7): 940-952.

10. Havel JJ, Chowell D, Chan TA. The evolving landscape of biomarkers for checkpoint inhibitor immunotherapy. Nat Rev Cancer. 2019. 19(3): 133-150.

11. Lee KH, Lee MS. Atezolizumab plus bevacizumab for unresectable hepatocellular carcinoma - Authors' replys. Lancet Oncol. 2020. 21(9): e413.

12. Devergne O, Birkenbach M, Kieff E. Epstein-Barr virus-induced gene 3 and the p35 subunit of interleukin 12 form a novel heterodimeric hematopoietin. Proc Natl Acad Sci U S A. 1997. 94(22): 12041-6.

13. Zhang J, Zhang $Y$, Wang Q, et al. Interleukin-35 in immune-related diseases: protection or destruction. Immunology. 2019. 157(1): 13-20.

14. Zhao Z, Chen $X$, Hao S, et al. Increased interleukin-35 expression in tumor-infiltrating lymphocytes correlates with poor prognosis in patients with breast cancer. Cytokine. 2017. 89: 76-81.

15. Pankratz S, Bittner S, Herrmann AM, et al. Human CD4+ HLA-G+ regulatory T cells are potent suppressors of graft-versus-host disease in vivo. FASEB J. 2014. 28(8): 3435-45.

16. Allan SE, Song-Zhao GX, Abraham T, McMurchy AN, Levings MK. Inducible reprogramming of human $T$ cells into Treg cells by a conditionally active form of FOXP3. Eur J Immunol. 2008. 38(12): 3282-9.

17. Collison LW, Workman CJ, Kuo TT, et al. The inhibitory cytokine IL-35 contributes to regulatory T-cell function. Nature. 2007. 450(7169): 566-9.

18. Lee CC, Lin JC, Hwang WL, et al. Macrophage-secreted interleukin-35 regulates cancer cell plasticity to facilitate metastatic colonization. Nat Commun. 2018. 9(1): 3763.

19. Guo H, Li B, Wang W, Zhao N, Gao H. Mesenchymal stem cells overexpressing IL-35: a novel immunosuppressive strategy and therapeutic target for inducing transplant tolerance. Stem Cell Res Ther. 2018. 9(1): 254.

20. Huang C, Li Z, Li N, et al. Interleukin 35 Expression Correlates With Microvessel Density in Pancreatic Ductal Adenocarcinoma, Recruits Monocytes, and Promotes Growth and Angiogenesis of Xenograft Tumors in Mice. Gastroenterology. 2018. 154(3): 675-688.

21. Fu YP, Yi Y, Cai $X Y$, et al. Overexpression of interleukin-35 associates with hepatocellular carcinoma aggressiveness and recurrence after curative resection. $\mathrm{Br} J$ Cancer. 2016. 114(7): 767-76. 
22. Zhang MX, Gan W, Jing CY, et al. Overexpression of interleukin-35 in intrahepatic cholangiocarcinoma is a prognostic indicator after curative resection. Cancer Sci. 2018. 109(4): 1195-1206.

23. Wang Z, Liu JQ, Liu Z, et al. Tumor-derived IL-35 promotes tumor growth by enhancing myeloid cell accumulation and angiogenesis. J Immunol. 2013. 190(5): 2415-23.

24. Nicholl MB, Ledgewood CL, Chen X, et al. IL-35 promotes pancreas cancer growth through enhancement of proliferation and inhibition of apoptosis: evidence for a role as an autocrine growth factor. Cytokine. 2014. 70(2): 126-33.

25. Huang C, Li N, Li Z, et al. Tumour-derived Interleukin 35 promotes pancreatic ductal adenocarcinoma cell extravasation and metastasis by inducing ICAM1 expression. Nat Commun. 2017. 8: 14035.

26. Ma Y, Chen L, Xie G, et al. Elevated level of interleukin-35 in colorectal cancer induces conversion of T cells into iTr35 by activating STAT1/STAT3. Oncotarget. 2016. 7(45): 73003-73015.

27. Turnis ME, Sawant DV, Szymczak-Workman AL, et al. Interleukin-35 Limits Anti-Tumor Immunity. Immunity. 2016. 44(2): 316-29.

28. Zou JM, Qin J, Li YC, et al. IL-35 induces N2 phenotype of neutrophils to promote tumor growth. Oncotarget. 2017. 8(20): 33501-33514.

29. Huang JL, Fu YP, Gan W, et al. Hepatic stellate cells promote the progression of hepatocellular carcinoma through microRNA-1246-RORa-Wnt/ $\beta$-Catenin axis. Cancer Lett. 2020. 476: 140-151.

30. Chandrasekar B, Mitchell DH, Colston JT, Freeman GL. Regulation of CCAAT/Enhancer binding protein, interleukin-6, interleukin-6 receptor, and gp130 expression during myocardial ischemia/reperfusion. Circulation. 1999. 99(3): 427-33.

31. Zhou SL, Hu ZQ, Zhou ZJ, et al. miR-28-5p-IL-34-macrophage feedback loop modulates hepatocellular carcinoma metastasis. Hepatology. 2016. 63(5): 1560-75.

32. Rotondo R, Barisione G, Mastracci L, et al. IL-8 induces exocytosis of arginase 1 by neutrophil polymorphonuclears in nonsmall cell lung cancer. Int J Cancer. 2009. 125(4): 887-93.

33. Liang S, Hoskins M, Dong C. Tumor cell extravasation mediated by leukocyte adhesion is shear rate dependent on IL-8 signaling. Mol Cell Biomech. 2010. 7(2): 77-91.

34. Szczerba BM, Castro-Giner F, Vetter M, et al. Neutrophils escort circulating tumour cells to enable cell cycle progression. Nature. 2019. 566(7745): 553-557.

35. Huh SJ, Liang S, Sharma A, Dong C, Robertson GP. Transiently entrapped circulating tumor cells interact with neutrophils to facilitate lung metastasis development. Cancer Res. 2010. 70(14): 6071-82.

36. Gordon-Weeks AN, Lim SY, Yuzhalin AE, et al. Neutrophils promote hepatic metastasis growth through fibroblast growth factor 2-dependent angiogenesis in mice. Hepatology. 2017. 65(6): 1920-1935.

37. Tang J, Shalabi A, Hubbard-Lucey VM. Comprehensive analysis of the clinical immuno-oncology landscape. Ann Oncol. 2018. 29(1): 84-91.

38. Schmidt EV. Developing combination strategies using PD-1 checkpoint inhibitors to treat cancer. Semin Immunopathol. 2019. 41(1): 21-30.

39. Zhou C, Zhang J. Immunotherapy-based combination strategies for treatment of gastrointestinal cancers: current status and future prospects. Front Med. 2019. 13(1): 12-23. 
40. Jia Y, Li X, Jiang T, et al. EGFR-targeted therapy alters the tumor microenvironment in EGFR-driven lung tumors: Implications for combination therapies. Int J Cancer. 2019. 145(5): 1432-1444.

41. Long L, Zhao C, Ozarina M, Zhao X, Yang J, Chen H. Targeting Immune Checkpoints in Lung Cancer: Current Landscape and Future Prospects. Clin Drug Investig. 2019. 39(4): 341-353.

42. Namikawa K, Yamazaki N. Targeted Therapy and Immunotherapy for Melanoma in Japan. Curr Treat Options Oncol. 2019. 20(1): 7.

43. He G, Zhang H, Zhou J, et al. Peritumoural neutrophils negatively regulate adaptive immunity via the PDL1/PD-1 signalling pathway in hepatocellular carcinoma. J Exp Clin Cancer Res. 2015. 34: 141.

\section{Tables}

Table 1. the correlation between clinicopathologic characters and IL35 


\begin{tabular}{|c|c|c|c|}
\hline \multirow[t]{2}{*}{ characters } & \multicolumn{3}{|c|}{ Expression of IL-35 } \\
\hline & Low & High & $P$ value \\
\hline Total patients & 210 & 150 & \\
\hline Gender, male/femeal & $113 / 97$ & $89 / 61$ & 0.298 \\
\hline Age, $<60 / \geq 60$ & $133 / 77$ & $107 / 43$ & 0.112 \\
\hline HBsAg, negative/positive & $24 / 186$ & $22 / 128$ & 0.364 \\
\hline $\mathrm{AFP},<400 / \geq 400 \mathrm{ng} / \mathrm{mL}$ & $116 / 94$ & $51 / 99$ & $<0.001$ \\
\hline $\mathrm{TBIL},<20 / \geq 20 \mu \mathrm{mol} / \mathrm{L}$ & $179 / 31$ & $122 / 28$ & 0.324 \\
\hline $\mathrm{AST},<45 / \geq 45 \mathrm{U} / \mathrm{L}$ & $127 / 83$ & $88 / 62$ & 0.73 \\
\hline $\mathrm{ALT},<50 / \geq 50 \mathrm{U} / \mathrm{L}$ & $171 / 39$ & $116 / 34$ & 0.341 \\
\hline ALB, $<35 / \geq 35 \mathrm{~g} / \mathrm{L}$ & $15 / 195$ & $5 / 145$ & 0.12 \\
\hline cirrhosis,no/yes & $55 / 155$ & $29 / 121$ & 0.129 \\
\hline tumor number,single/multiple & $177 / 33$ & $33 / 35$ & 0.069 \\
\hline tumor throbus,no/yes & $204 / 6$ & $110 / 40$ & $<0.001$ \\
\hline tumor capsule,no/yes & $59 / 151$ & $49 / 101$ & 0.351 \\
\hline tumor size, $<5 / \geq 5 \mathrm{~cm}$ & $138 / 72$ & $80 / 70$ & 0.018 \\
\hline LNM,no/yes & $205 / 5$ & $145 / 5$ & 0.588 \\
\hline MVI, no/yes & $303 / 83$ & $27 / 27$ & $<0.001$ \\
\hline differentiation, I-II/III-IV & $140 / 70$ & $75 / 75$ & 0.001 \\
\hline Counts of $\mathrm{CD} 34^{+}$cells $\mathbb{\text { low}} / \mathrm{high} \rrbracket$ & $158 / 52$ & $92 / 58$ & 0.005 \\
\hline Counts of Treg cells $\varangle$ low/high $\rrbracket$ & $134 / 76$ & $90 / 60$ & 0.462 \\
\hline Counts of $C D 8^{+} T$ cells $\rrbracket l o w / h i g h \rrbracket$ & $120 / 90$ & $112 / 38$ & 0.001 \\
\hline Counts of $\mathrm{CD} 4^{+} \mathrm{T}$ cells $\rrbracket$ low/high $\rrbracket$ & $181 / 29$ & $130 / 20$ & 0.897 \\
\hline Counts of $\mathrm{CD} 66 \mathrm{~b}^{+}$cells $\mathbb{\text { low }} / \mathrm{high} \rrbracket$ & $152 / 58$ & $66 / 84$ & $<0.001$ \\
\hline Counts of $\mathrm{CD} 68^{+}$cells $\mathbb{\text { low}} / \mathrm{high} \rrbracket$ & $138 / 72$ & $89 / 61$ & 0.216 \\
\hline$B C L C$ stage $\triangle A / B / C \rrbracket$ & $132 / 72 / 6$ & $64 / 46 / 40$ & $<0.001$ \\
\hline
\end{tabular}

APF, alpha fetal protein, LNM, lymph node metastasis, MVI, microvascular invasion,BCLC stage, Barcelona Clinic Liver Cancer staging system 
Table2.univariate and multivariate analyses for OS and RFS in the cohort 


\begin{tabular}{|c|c|c|c|c|c|c|}
\hline \multirow{3}{*}{ characters } & \multicolumn{3}{|l|}{ OS } & \multicolumn{3}{|l|}{ RFS } \\
\hline & univariate & multivariate & $\mathrm{HR}$ & univariate & multivariate & $\mathrm{HR}$ \\
\hline & $P$ value & Pvalue & $(95 \% \mathrm{Cl})$ & $P$ value & $P$ value & $(95 \% \mathrm{Cl})$ \\
\hline $\begin{array}{l}\text { Gender, } \\
\text { male/femeal }\end{array}$ & 0.559 & NA & NA & 0.568 & NA & NA \\
\hline Age, $<60 / \geq 60$ & 0.266 & NA & NA & 0.237 & NA & NA \\
\hline $\begin{array}{l}\text { HBsAg, } \\
\text { negative/positive }\end{array}$ & 0.598 & NA & NA & 0.394 & NA & NA \\
\hline $\begin{array}{l}\text { TBIL, }<20 / \geq 20 \\
\mu \mathrm{mol} / \mathrm{L}\end{array}$ & 0.528 & NA & NA & 0.921 & NA & NA \\
\hline $\mathrm{ALT},<50 / \geq 50 \mathrm{U} / \mathrm{L}$ & 0.716 & NA & NA & 0.440 & NA & NA \\
\hline$A L B,<35 / \geq 35 \mathrm{~g} / \mathrm{L}$ & 0.052 & NA & NA & 0.075 & NA & NA \\
\hline $\begin{array}{l}\mathrm{AFP},<400 / \geq 400 \\
\mathrm{ng} / \mathrm{mL}\end{array}$ & 0.021 & NS & NS & 0.001 & NS & NS \\
\hline $\begin{array}{l}\mathrm{AST},<45 / \geq 45 \\
\mathrm{U} / \mathrm{L}\end{array}$ & 0.094 & NA & NA & 0.004 & 0.007 & $\begin{array}{l}1.565(1.131- \\
2.166)\end{array}$ \\
\hline $\begin{array}{l}\text { tumor size },<5 / \\
\geq 5 \mathrm{~cm}\end{array}$ & {$[0.001$} & 0.012 & $\begin{array}{l}1.634(1.113- \\
2.399)\end{array}$ & {$[0.001$} & NS & NS \\
\hline $\begin{array}{l}\text { tumor number, } \\
\text { single/multiple }\end{array}$ & {$[0.001$} & 0.016 & $\begin{array}{l}1.643(1.097- \\
2.460)\end{array}$ & {$[0.001$} & NS & NS \\
\hline $\begin{array}{l}\text { tumor } \\
\text { capsule,no/yes }\end{array}$ & 0.158 & NA & NA & 0.029 & NS & NS \\
\hline MVI, no/yes & {$[0.001$} & NS & NS & {$[0.001$} & NS & NS \\
\hline $\begin{array}{l}\text { tumor } \\
\text { throbus,no/yes }\end{array}$ & 00.001 & 0004 & $\begin{array}{l}2.073(1.262- \\
3.403)\end{array}$ & {$[0.001$} & $\square 0.001$ & $\begin{array}{l}2.507(1.610- \\
3.906)\end{array}$ \\
\hline $\begin{array}{l}\text { differentiation, I- } \\
\text { II/III-IV }\end{array}$ & 0.015 & NS & NS & 0.041 & NS & NS \\
\hline LNM,no/yes & 0.023 & NS & NS & 0.059 & NA & NA \\
\hline cirrhosis,no/yes & 0.071 & NA & NA & 0.010 & NS & NS \\
\hline $\begin{array}{l}\text { Expression of IL- } \\
35\end{array}$ & 00.001 & 0.035 & $\begin{array}{l}1.947(1.046- \\
3.624)\end{array}$ & प0.001 & 0.001 & $\begin{array}{l}2.442(1.459- \\
4.088)\end{array}$ \\
\hline $\begin{array}{l}\text { Counts of } \mathrm{CD} 34^{+} \\
\text {cells } \\
\text { (low/high } \rrbracket\end{array}$ & 00.001 & {$[0.001$} & $\begin{array}{l}3.919(2.589- \\
5.932)\end{array}$ & {$[0.001$} & 00.001 & $\begin{array}{l}2.329(1.658- \\
3.272)\end{array}$ \\
\hline $\begin{array}{l}\text { Counts of Treg } \\
\text { cells }\end{array}$ & 0.972 & NA & NA & 0.974 & NA & NA \\
\hline 『low/high $\rrbracket$ & & & & & & \\
\hline
\end{tabular}




\begin{tabular}{|c|c|c|c|c|c|c|}
\hline $\begin{array}{l}\text { Counts of } \mathrm{CD}^{+} \mathrm{T} \\
\text { cells }\end{array}$ & 0.053 & NA & NA & 0.027 & NS & NS \\
\hline \multicolumn{7}{|l|}{ 『low/high $\rrbracket$} \\
\hline $\begin{array}{l}\text { Counts of } \mathrm{CD} 4^{+} \mathrm{T} \\
\text { cells }\end{array}$ & 0.770 & NA & NA & 0.533 & NA & NA \\
\hline \multicolumn{7}{|l|}{ 邓low/high $\rrbracket$} \\
\hline $\begin{array}{l}\text { Counts of } \\
{\text { CD } 66 b^{+} \text {cells }}^{-1}\end{array}$ & $\square 0.001$ & 0.022 & $\begin{array}{l}1.625(1.074- \\
2.460)\end{array}$ & {$[0.001$} & 0.037 & $\begin{array}{l}1.420(1.022- \\
1.974)\end{array}$ \\
\hline \multicolumn{7}{|l|}{ 『low/high $\rrbracket$} \\
\hline $\begin{array}{l}\text { Counts of } \\
\text { CD68 } 8^{+} \text {cells }\end{array}$ & {$[0.001$} & NS & NS & {$[0.001$} & NS & NS \\
\hline \multicolumn{7}{|l|}{ 『low/high $\rrbracket$} \\
\hline $\begin{array}{l}\text { BCLC stage } \\
\text { (A/B/CD }\end{array}$ & {$[0.001$} & {$[0.001$} & $\begin{array}{l}2.205(1.709- \\
2.844)\end{array}$ & {$[0.001$} & {$[0.001$} & $\begin{array}{l}1.911(1.539- \\
2.374)\end{array}$ \\
\hline
\end{tabular}

Abbreviations $₫ \mathrm{APF}=$ alpha fetal protein;TBIL=total bilirubin,ALB=albumin,BCLC=Barcelona Clinic Liver Cancer staging system, NA囚not applicable, NS, not significant

Figures 
A

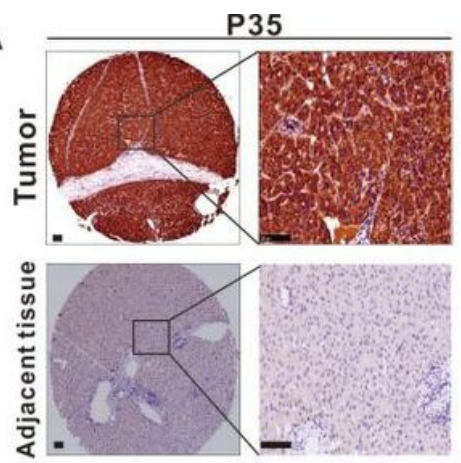

B

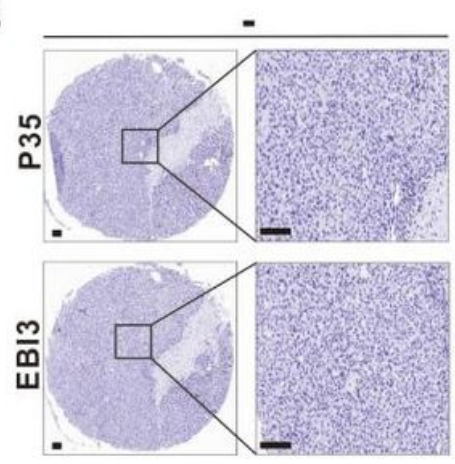

C
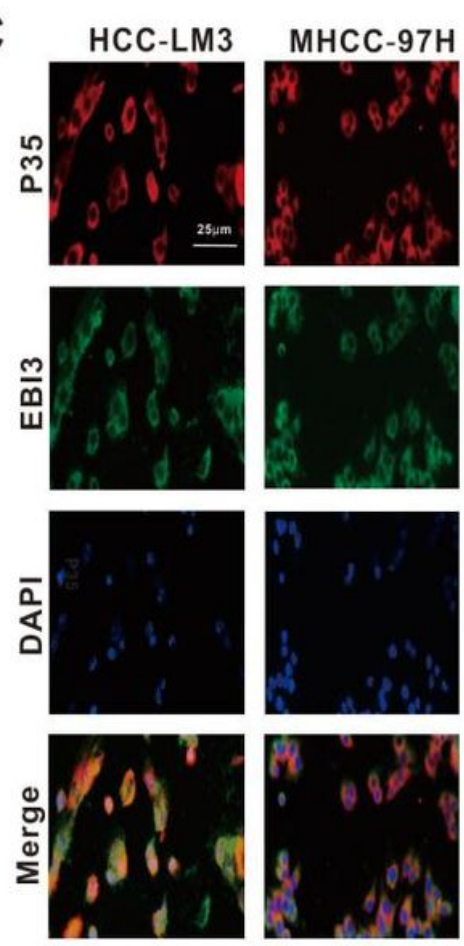
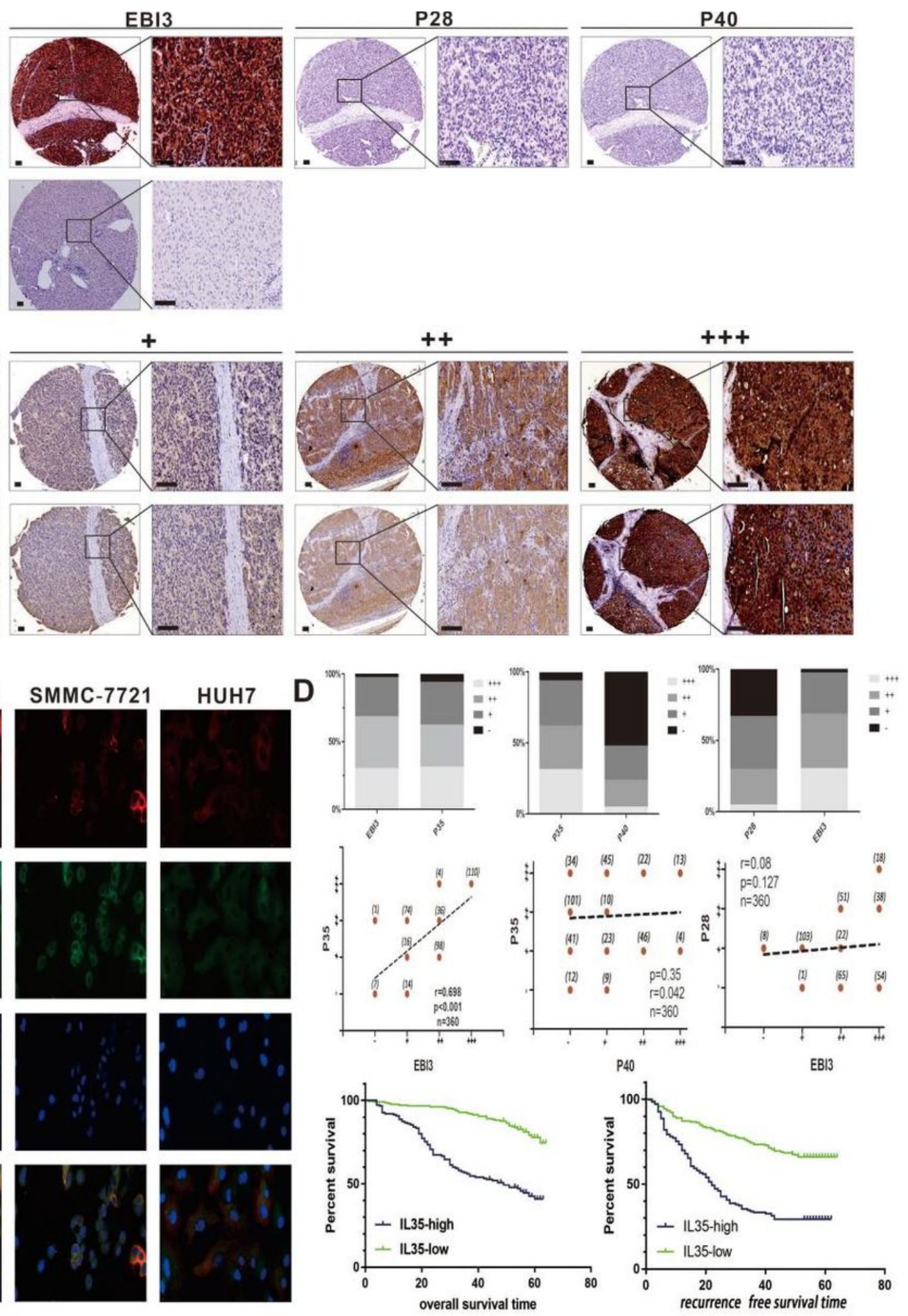

Figure 1

High expression of IL-35 confers a poor prognosis in HCC patients. Serial sections of HCC tissue microarray (TMA) were used to explore the expression levels of the two subunits of the IL-35 ligand: EBI3 and P35. As EBI3 forms IL-27 with P28 and P35 forms IL-12 with P40, we also examined the expression levels of P28 and P40 in consecutive tumor tissue. A. a significant higher expression of P35 and EBI3 in HCC tumor were found than that of adjacent normal tissue. And the expression levels were much higher than that of P40 and P28 In consecutive sections of HCC tissue. B.The expression levels of P35 and EBI3 are highly consistent. And these are the representative pictures of P35 and EBI3 from low to high depending on the expression level $(-,+,++,+++)$. 
C. Cell fluorescent microscopy images demonstrating that the expression of P35 (red), EBI3 (green) were higher in HCC tumor cells HCC-LM3 and MHCC-97H than that of SMMC-7721 and HUH7. D. The distribution bar chart of P35, EBI3, P28, P40 in 360 HCC patients. and, staining extent correlation among them. We have also illustrated the Kaplan-Meier survival analysis of OS and RFS according to different IL-35 levels.

A
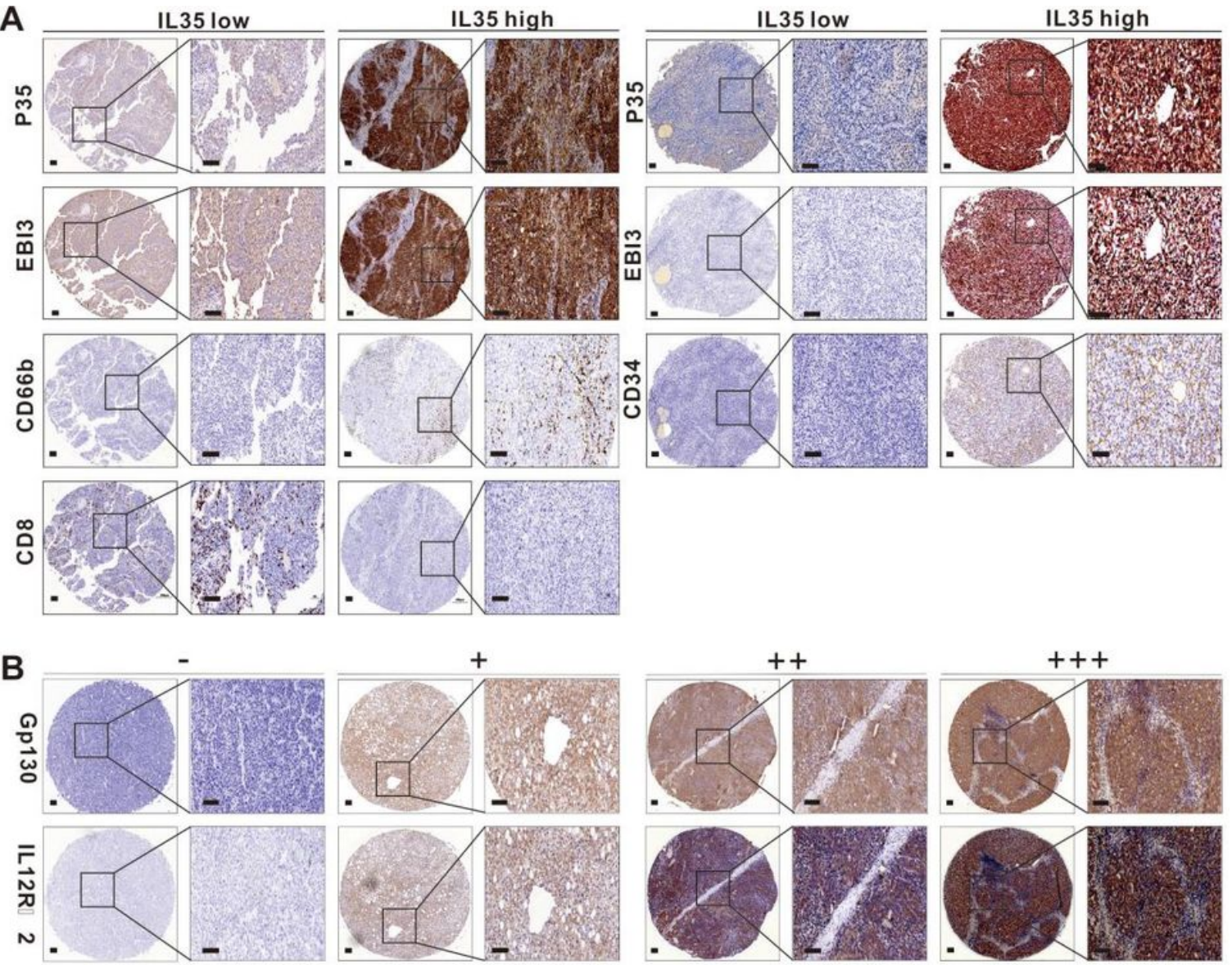

C
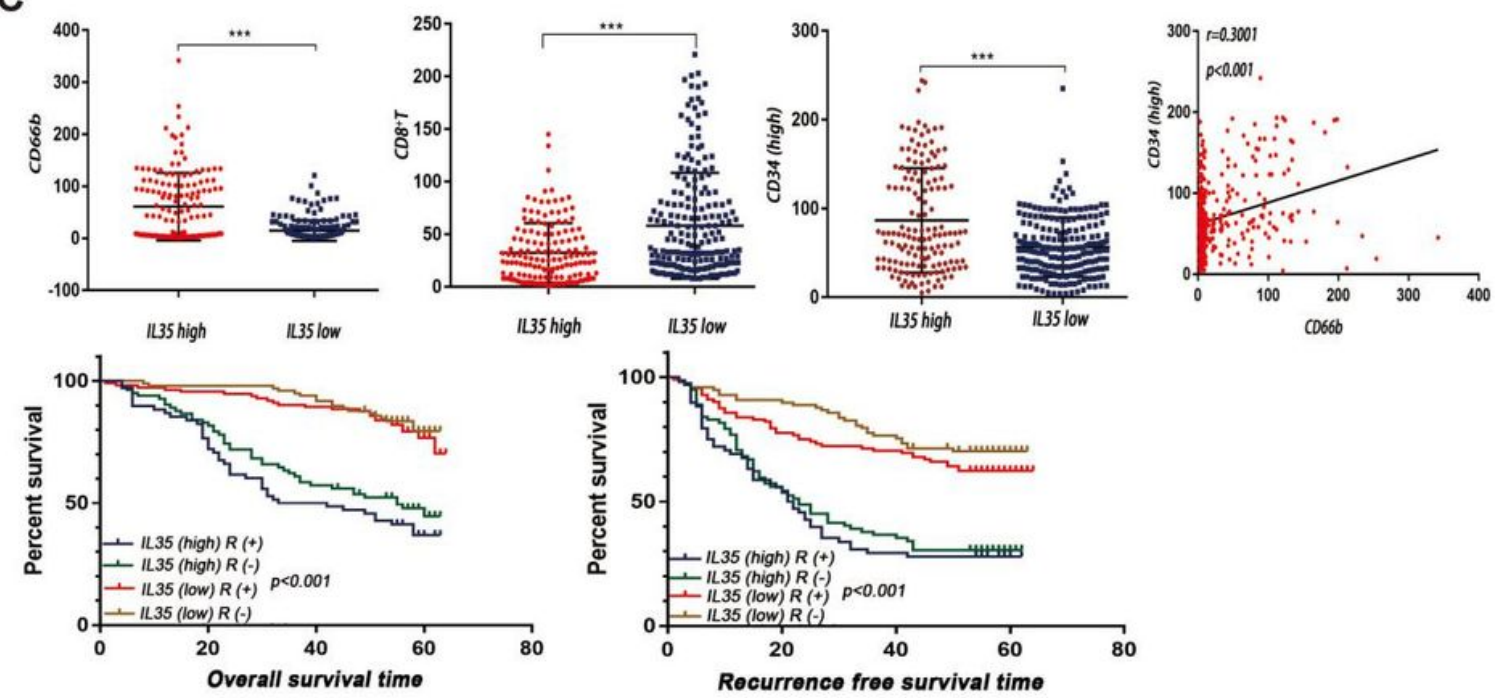

Figure 2

Higher IL-35 expression in HCC correlation with the infiltration of CD66b cell , formation of MVD and decrease of $\mathrm{CD} 8+\mathrm{T}$ cell. A.The representative images of immunohistochemistry (IHC) staining for P35, EBI3, CD66b, $\mathrm{CD} 8+\mathrm{T}, \mathrm{CD} 34$. According to the expression of P35 and EBI3, patients were distributed into high IL-35 group 
and low IL-35 group. B.The expression levels of Gp130 and IL12-Rß2 are also consistent. the representative pictures of Gp130 and IL12-Rß2 from low to high depending on the expression level (absent -, low +, middle ++, high +++) were illustrated. C.Quantification of MVD, CD66b+ , CD8+ T counts per fields were illustrated. And pearson correlation analysis of CD34+ and CD66b+ were carried out. Kaplan-Meier survival analysis of OS and RFS based on the corporation of IL-35 expression and IL-35 receptors were performed.
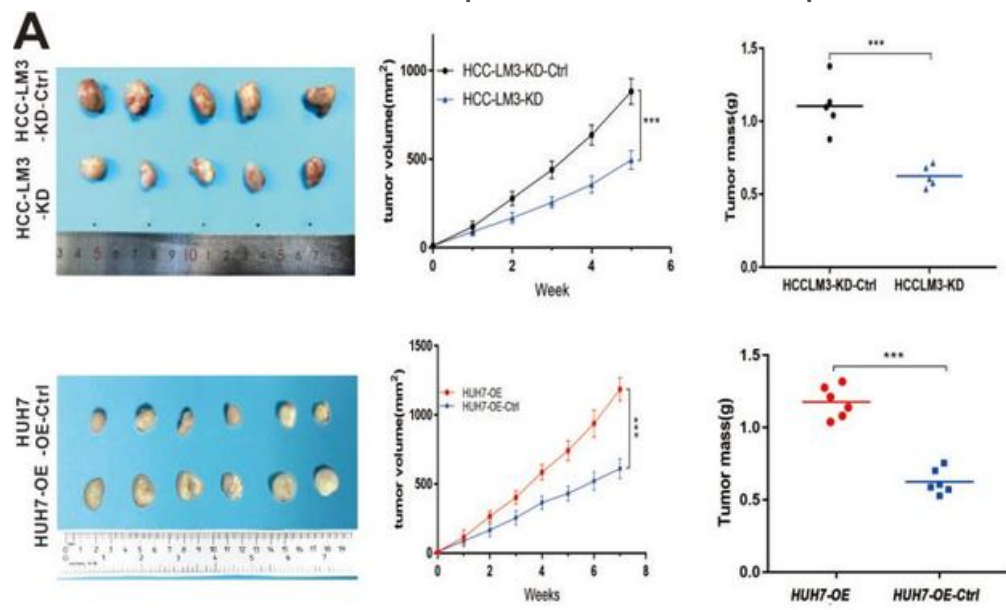

B
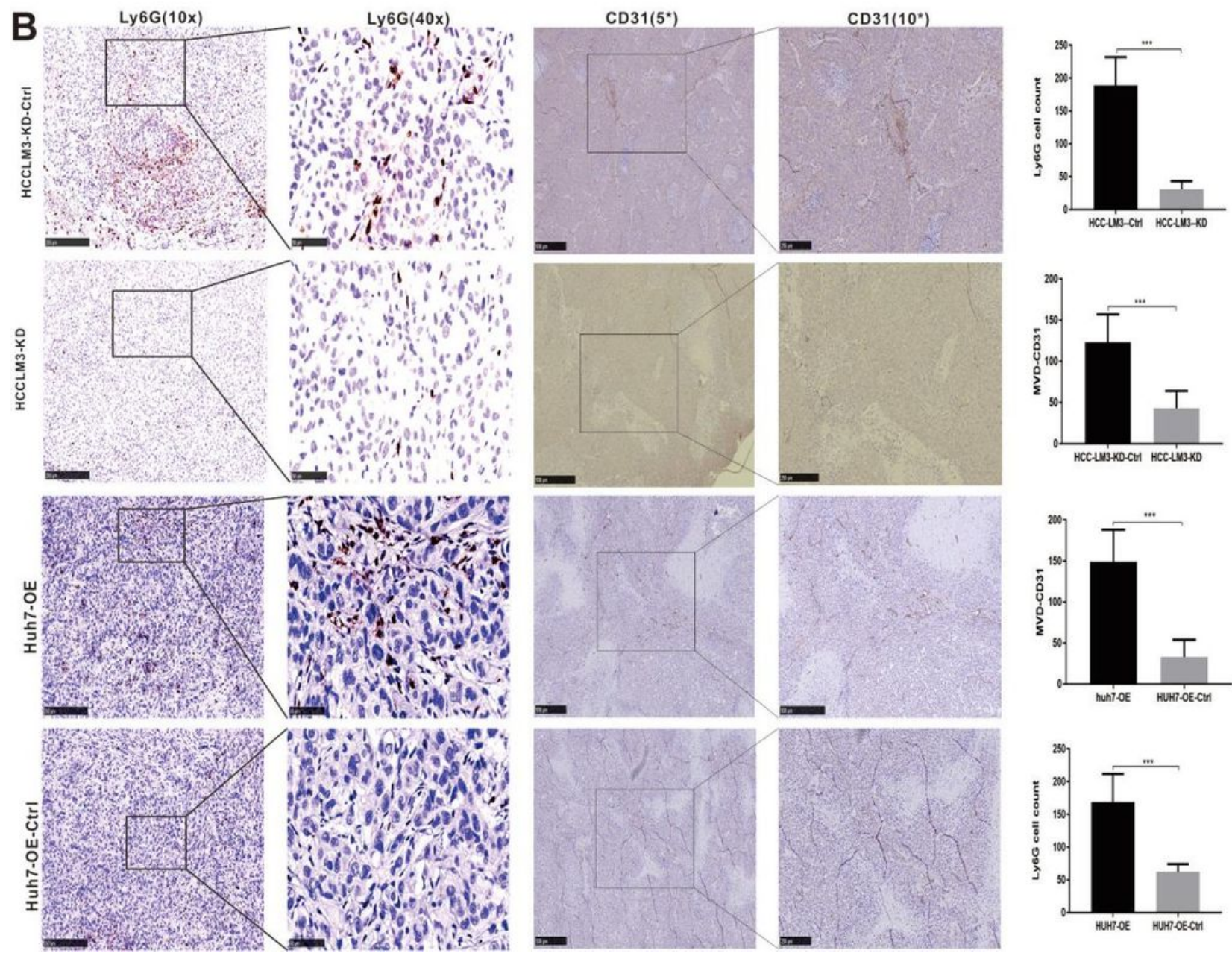

Figure 3

Overexpression of IL-35 significantly promoted HCC neovascularization and progression and recruited neutrophile in nude mouse models. A. Knockdown IL-35 in HCC-LM3 cell line significantly slow down the 
progression of HCC, Meanwhile overexpression of IL-35 would facilitate tumor growth in vivo experiments of nude mouse models. B. IL-35 increased intratumoral Ly6G+ neutrophile infiltration and MVD in nude mouse models.
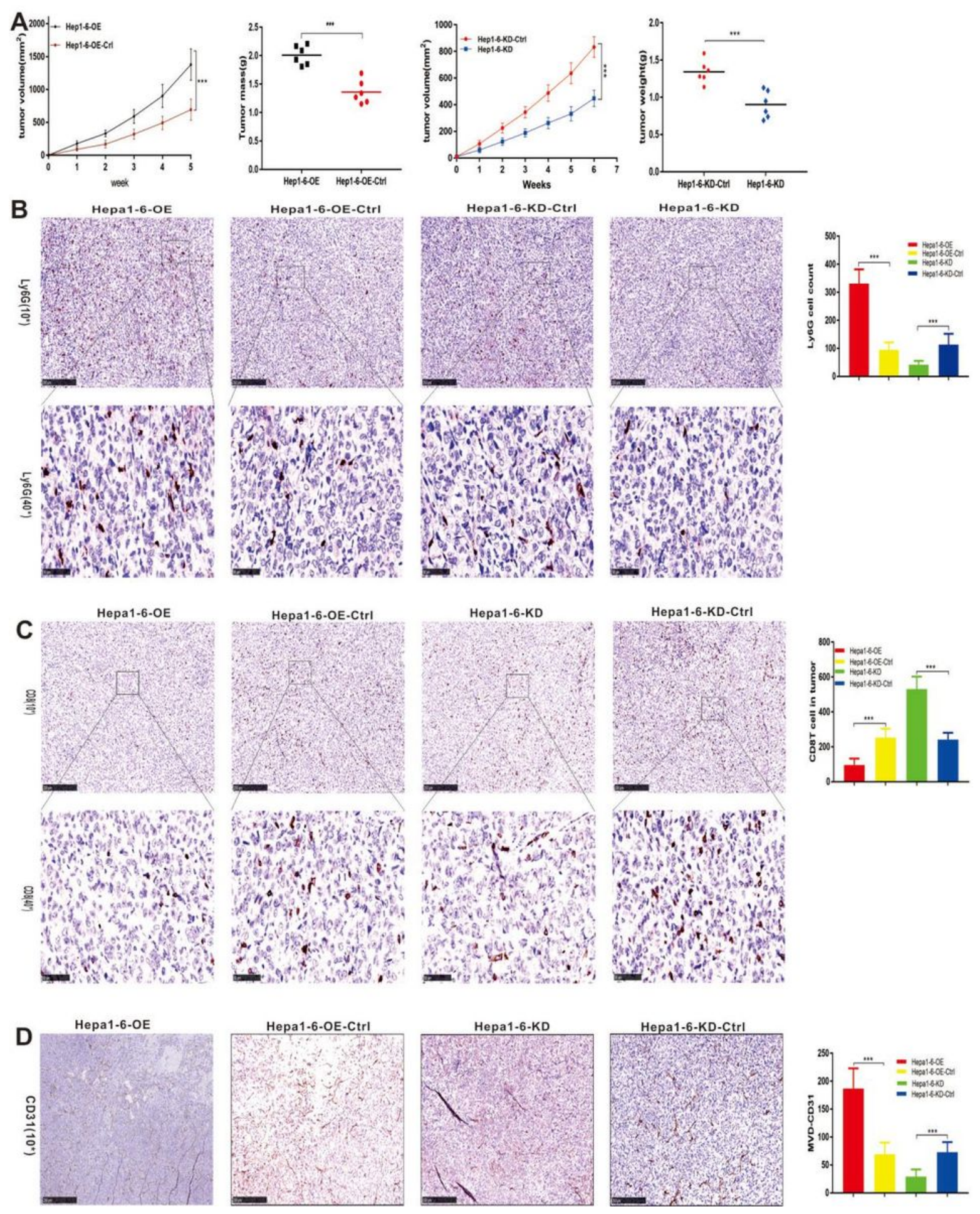

\section{Figure 4}

Overexpression of IL-35 significantly promoted HCC neovascularization and progression and recruited Ly6G+ neutrophile. but, influence the infiltration of CD8+T cell . A. Knockdown IL-35 in HCC cell line significantly slow down the progression of HCC, and increase IL-35 would facilitate tumor growth in vivo experiments.B.IL-35 increased intratumoral Ly6G+ neutrophile infiltration.C.IL-35 influence the infiltrated number of CD8+T cells. 
D.Overexpression of IL-35 increased neovascularization in tumor, and MVD would decreased when IL-35 were knocked down.
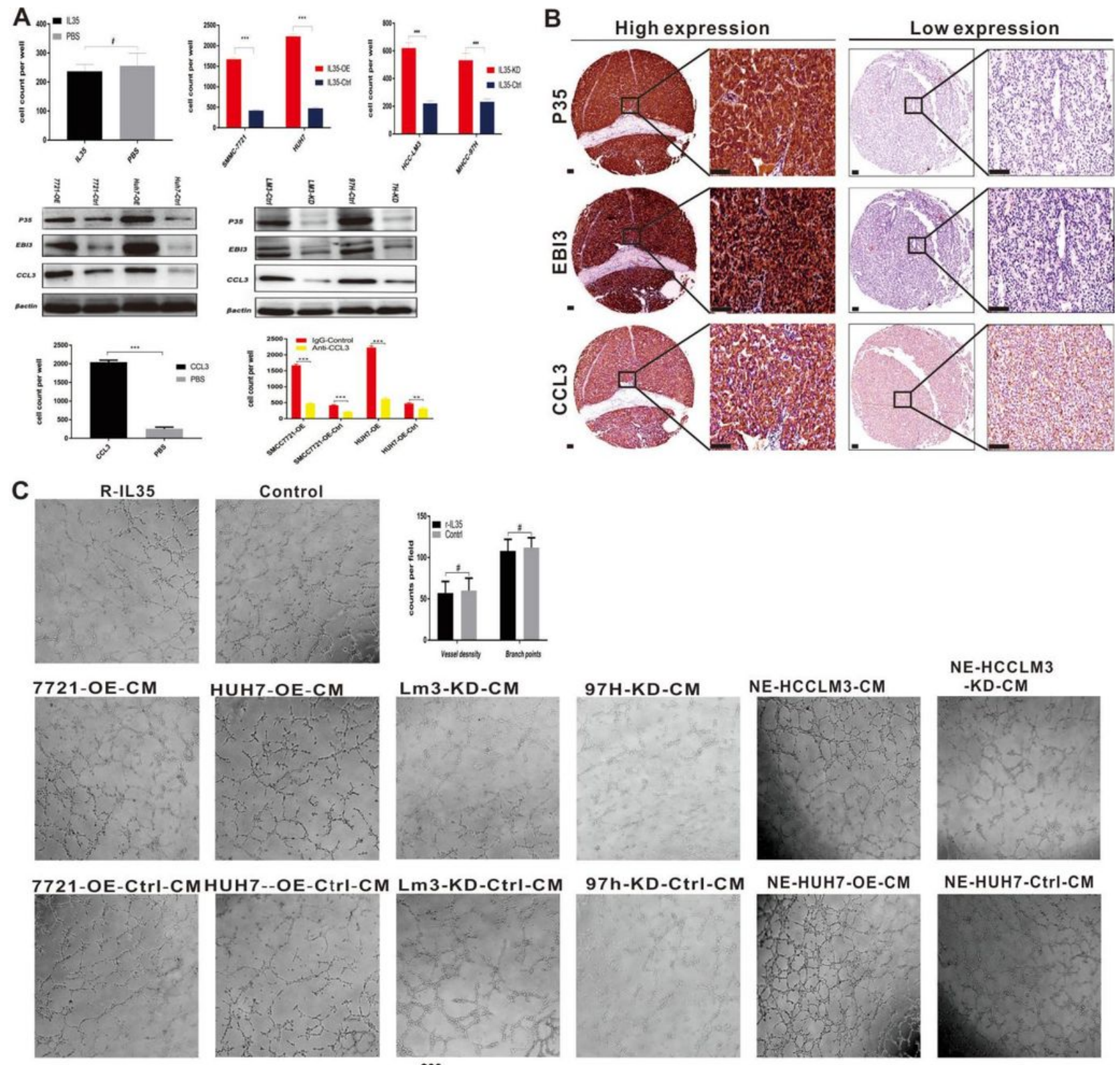

97h-KD-Ctrl-CM
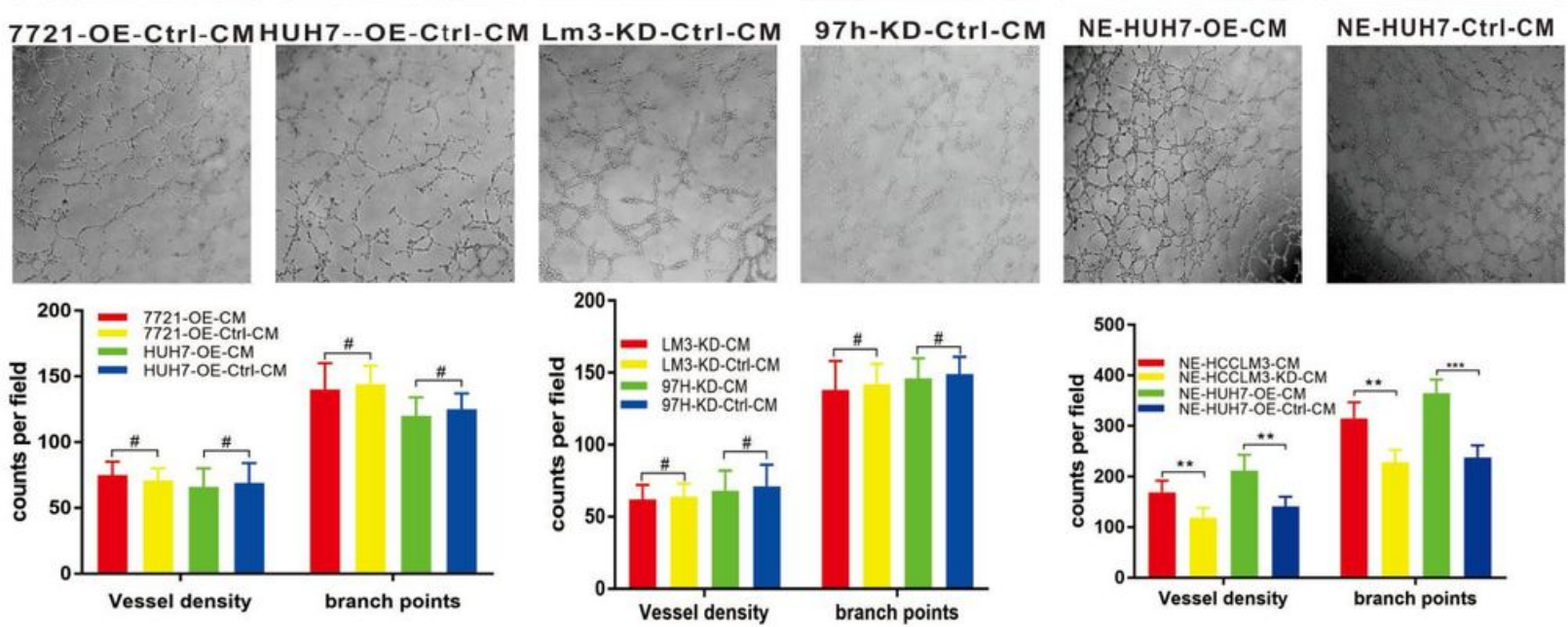

\section{Figure 5}

IL-35 might recruited neutrophiles by increase the expression of CCL3, and affiliated tuber formation of HUVEC by stimulating neutrophiles. A. IL-35 recruited neutrophils by regulating the expression of CCL3 in HCC cell line. B.The expression of IL-35 are highly correlated wiht CCL3 in HCC patient tissues. C.IL-35 affiliated neutrophil tuber formation ability through increase the tube density and the branch points. 

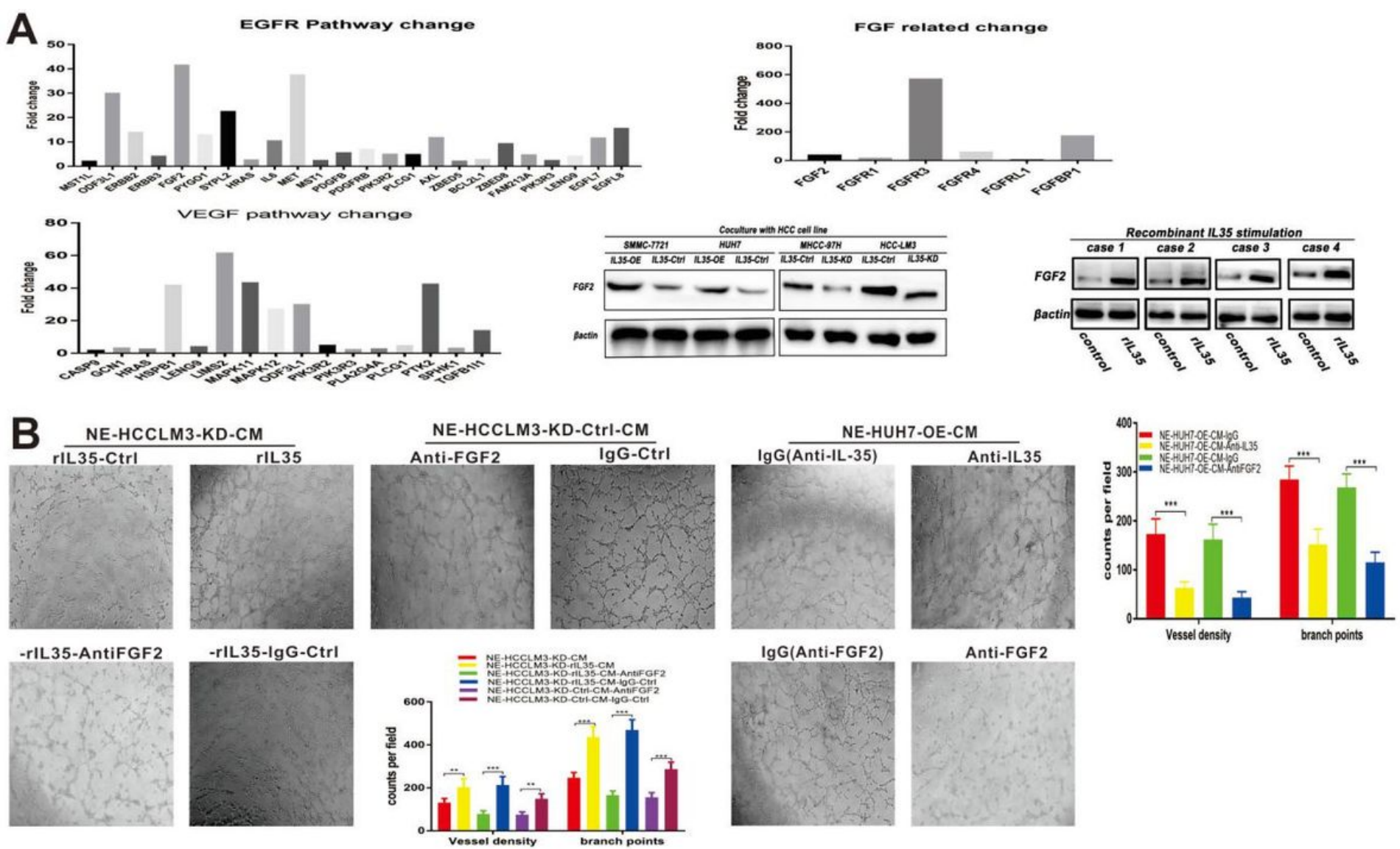

\section{Figure 6}

The tuber formation capable of neutrophils were activated in a FGF2 depended manner while it was stimulated by IL-35. A. Neutrophils's proangiogenetic capability were activated by increasing the secretion of FGF2. B.Anti-FGF2 antibody were used to blocked IL-35 mediated neutrophils' proangiogenetic capability. And blocking IL-35 with IL-35 antibody also could decrease the vessel density and branch points in co-culture system of HCC and neutrophiles. 


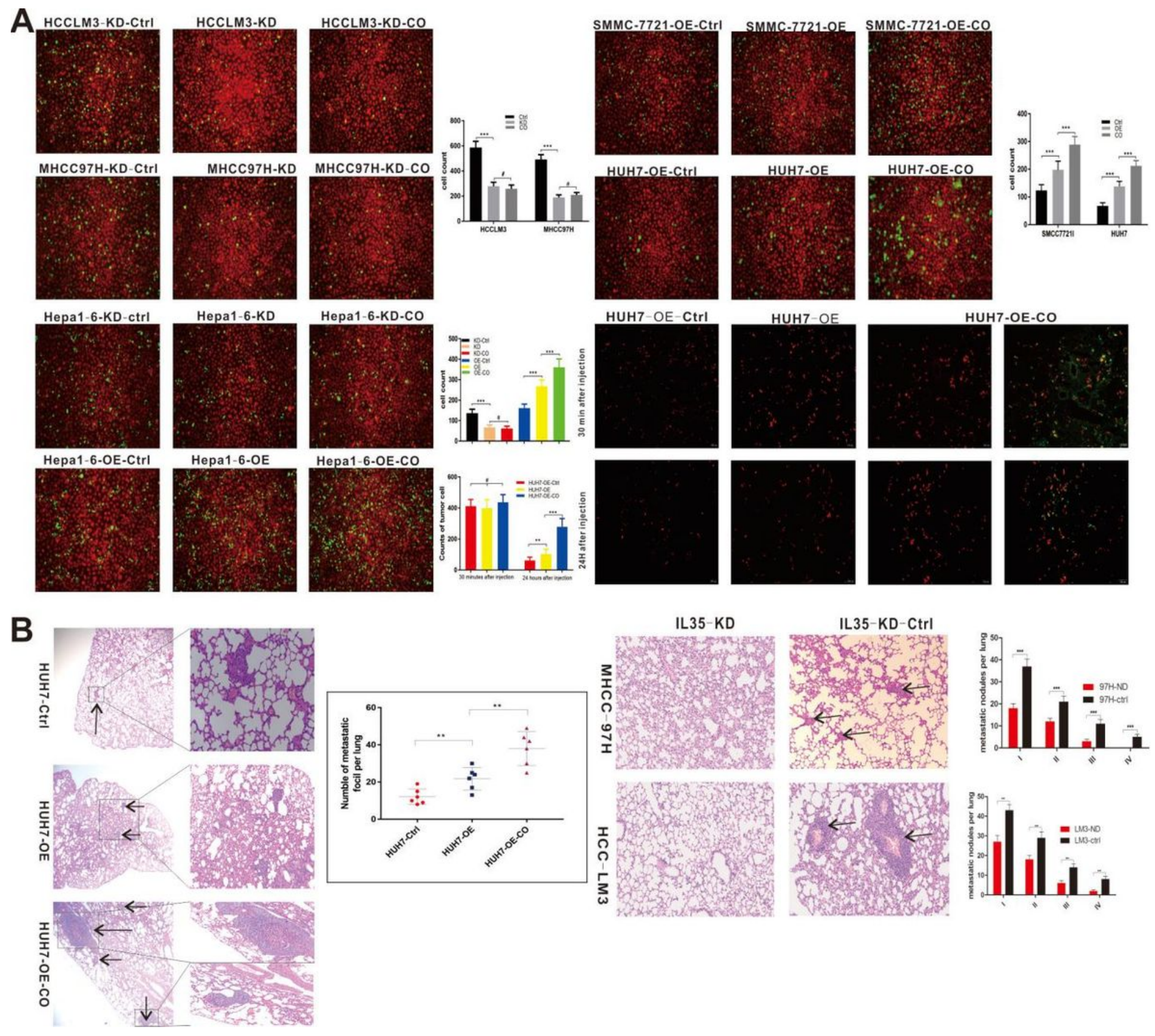

Figure 7

IL-35 affiliated the adhesion between HCC tumor cell and HUVEC, and promoted the pulmonary metastasis in vivo. A. More HCC were found to adhere the surface of HUVEC. In vivo experiment the remaining tumor cells in lung tissue were observed by fluorescence tracer. We found that there was no significant difference in the retention of tumor cells in each group at 30 minutes, but there was a significant difference in the number of tumor cells stranded in lung tissue 24 hours later. B. HE staining were used to count the number of pulmonary metastatic nodules in lung metastasis model. Significantly increased metastatic nodules were found in overexpression co-inject group. Meanwhile, we found that the lung metastasis rate decreased significantly when IL-35 was knocking down. 


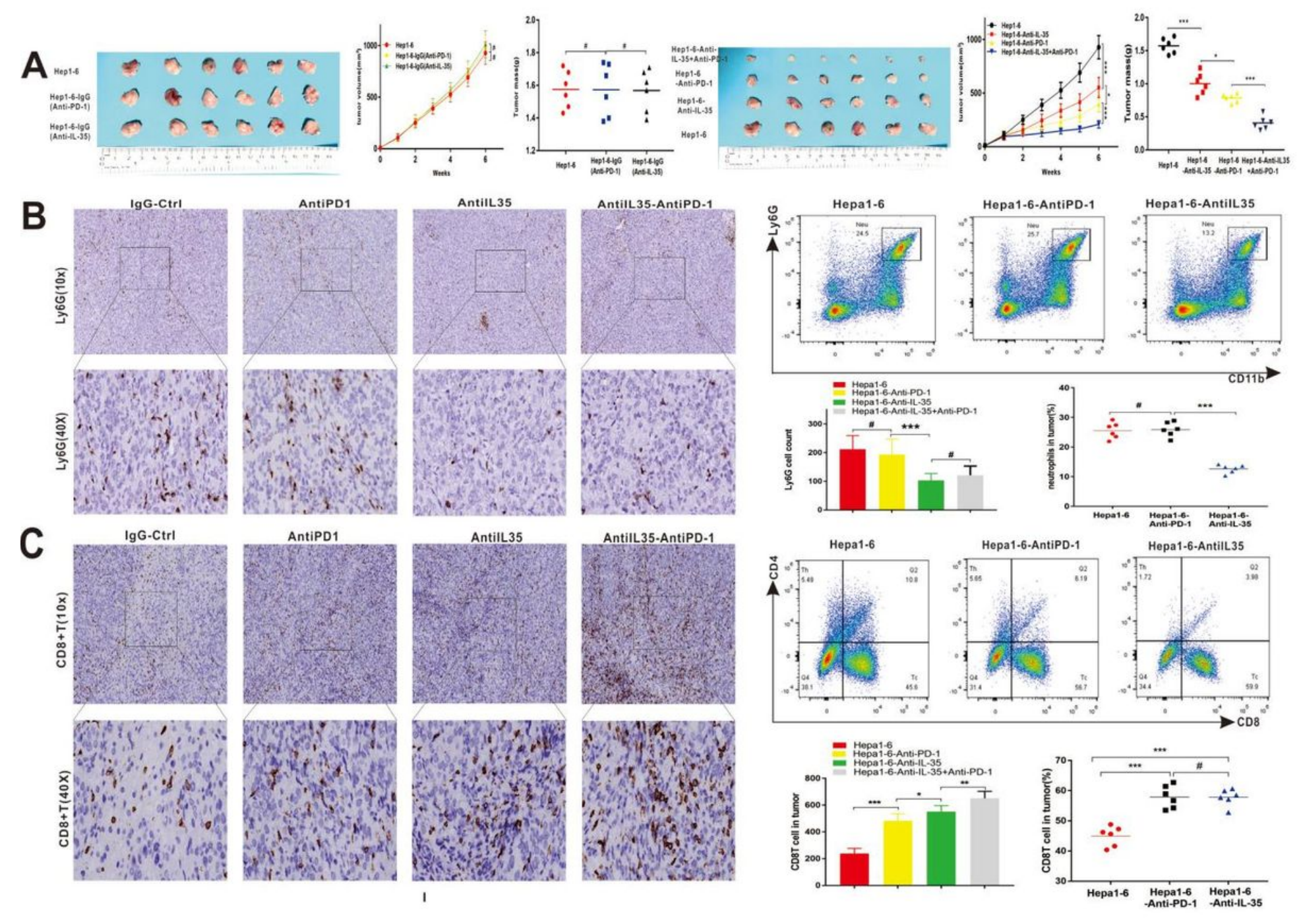

\section{Figure 8}

AntilL-35 antibody significantly enhance the therapy efficiency of PD-1 antibody in subcutaneous tumor model of immunocompetent mice recipients bearing Hepa1-6 cells A. Either antilL-35 antibody or antiPD-1 antibody could apparently slow down HCC progression in vivo treatment experiments. Combination therapy with both antibodies have dramatic and more durable responses for the treatment of HCC. B. IHC and flow cytometric plot were utilized to explore the infiltration of immune cells of these tumors tissue. Anti-PD-1 antibody has no effect on the infiltration of Ly6G+ neutrophiles, but significant difference were catch in combined treatment group and antilL-35 group. There were less infiltration of neutrophils among these two groups. C. Interesting we also found that CD8+T cell were also significant increase in both PD-1 antidy group and combined treatment group.

\section{Supplementary Files}

This is a list of supplementary files associated with this preprint. Click to download.

- Figs1toS4.docx 